\title{
Backward Stochastic Differential Equations and Dirichlet Problems of Semilinear Elliptic Operators with Singular Coefficients
}

\author{
Saisai Yang ${ }^{1}$ - Tusheng Zhang ${ }^{2}$
}

Received: 30 March 2017 / Accepted: 15 September 2017 / Published online: 16 November 2017

(C) The Author(s) 2017. This article is an open access publication

\begin{abstract}
In this paper, we prove that there exists a unique solution to the Dirichlet boundary value problem for a general class of semilinear second order elliptic differential operators which do not necessarily have the maximum principle and are non-symmetric in general. Our method is probabilistic. It turns out that we need to solve a class of backward stochastic differential equations with singular coefficients, which is of independent interest itself. The theory of Dirichlet forms also plays an important role.
\end{abstract}

Keywords Dirichlet boundary value problem - Semilinear second order elliptic differential equations - Dirichlet forms - Backward stochastic differential equations

Mathematics Subject Classifications (2010) Primary 60H60 • Secondary 60H30, 31J25

\section{Introduction}

In this paper, we will use probabilistic methods to solve the Dirichlet boundary value problem for the semilinear second order elliptic partial differential equations (PDEs) of the following form:

$$
\begin{cases}\mathcal{A} u(x)=-f(x, u(x)), & \forall x \in D \\ \left.u(x)\right|_{\partial D}=\varphi, & \forall x \in \partial D\end{cases}
$$

Tusheng Zhang

Tusheng.Zhang@manchester.ac.uk

Saisai Yang

yangss@mail.ustc.edu.cn

1 School of Mathematical Sciences, University of Science, Technology of China,

Hefei, 230026, China

2 School of Mathematics, University of Manchester, Oxford Road, Manchester, M13 9PL, UK 
where $D$ is a bounded domain in $\mathbb{R}^{d}, f(\cdot, \cdot)$ is a nonlinear function and $\varphi \in C(\partial D)$. The operator $\mathcal{A}$ is given by

$$
\mathcal{A} u=\frac{1}{2} \sum_{i, j=1}^{d} \frac{\partial}{\partial x_{i}}\left(a_{i j}(x) \frac{\partial u}{\partial x_{j}}\right)+\sum_{i=1}^{d} b_{i}(x) \frac{\partial u}{\partial x_{i}}-“ \operatorname{div}(\hat{b} u) "+q(x) u,
$$

where $a(x)=\left(a_{i j}(x)\right)_{1 \leq i, j \leq d}$ is a Borel measurable, (not necessarily symmetric) matrixvalued function on $\mathbb{R}^{d}$ satisfying

$$
\lambda|\xi|^{2} \leq \sum_{i, j=1}^{d} a_{i j}(x) \xi_{i} \xi_{j} \quad \forall \xi \in \mathbb{R}^{d}, x \in \mathbb{R}^{d}
$$

and

$$
a_{i j}(x) \leq \frac{1}{\lambda} \quad \forall x \in \mathbb{R}^{d}, 1 \leq i, j \leq d
$$

for some constant $0<\lambda \leq 1 ; b=\left(b_{1}, \ldots, b_{d}\right)^{*}, \hat{b}=\left(\hat{b}_{1}, \ldots, \hat{b}_{d}\right)^{*}$ and $q$ are Borel measurable functions and satisfy $|b|^{2} I_{D} \in L^{p}(D ; d x),|\hat{b}|^{2} I_{D} \in L^{p}(D ; d x)$ and $q I_{D} \in$ $L^{p}(D ; d x)$ for some constant $p>\frac{d}{2}$. Here $*$ stands for the transpose of a vector or matrix.

There is a big literature regarding the probabilistic approaches of solving boundary value problems. The pioneering work is traced back to Kukutani [12] who used Brownian motion to represent the solution of the classical Dirichlet problem for Laplacian operators. In the linear case, i.e. $f(\cdot, \cdot)=0$, if $\hat{b}=0, q \leq 0$, the problem (1.1) is solved by the Feynman-Kac formula

$$
u(x)=E_{x}\left[e^{\int_{0}^{\tau} D} q(X(s)) d s \varphi\left(X_{\tau_{D}}\right)\right],
$$

where $X=\left(\Omega, \mathcal{F}, \mathcal{F}_{t}, X_{t}, P_{x}, x \in \mathbb{R}^{d}\right)$ is the diffusion process associated with the generator $L_{1}$ given by

$$
L_{1} u=\frac{1}{2} \sum_{i, j=1}^{d} \frac{\partial}{\partial x_{i}}\left(a_{i j}(x) \frac{\partial u}{\partial x_{j}}\right)+\sum_{i=1}^{d} b_{i}(x) \frac{\partial u}{\partial x_{i}}
$$

and $\tau_{D}$ is the first exit time of $X$ from $D$. We refer the readers to Chen and Zhao [8] for details. The first result on probabilistic interpretation of viscosity solutions of semilinear parabolic PDEs was obtained by Peng in [17] and [16], through the backward stochastic differential equations, which is different from the weak solution considered in this paper.

Since $\hat{b}$ is merely measurable, some of the coefficients of the operator $\mathcal{A}$ are very singular (just distributions). When $\hat{b} \neq 0, f(\cdot, \cdot) \neq 0$ and the matrix $a(x)$ is symmetric, the boundary value problem (1.1) was considered by Zhang [18]. The time reversal of symmetric Markov processes and the backward stochastic differential equations (BSDEs) with random terminal time play an important role in [18]. When $a$ is non-symmetric and $f(\cdot, \cdot)=0$ (i.e. the linear case), Chen, Sun and Zhang [5] obtained a probabilistic representation of the solution to problem (1.1) under the condition that

$$
q-\operatorname{div} \hat{b} \leq g
$$

in the sense of distribution for a sufficient small non-negative function $g \in L^{p}(D)$.

In this paper, we consider the weak/Soblev solution (see the definition in the next section) of the semilinear problem (1.1). We will show that there exists a unique, continuous weak solution to the Dirichlet value problem (1.1) under appropriate conditions. We will use the h-transform method to tackle the singular term " $\operatorname{div}(\hat{b} \cdot)$ ". To solve the semilinear boundary 
value problem (1.1), we first produce a candidate to the solution by appealing to the theory of BSDEs. More precisely, we will solve a class of BSDEs with very singular coefficients and random terminal time. The BSDEs are driven by the martingale part of a diffusion process. The study of this class of BSDEs is of independent interest. It turns out that the classical $L^{2}$ setting of BSDEs is not suitable here. We have to work in the framework of $L^{1}$ and deal with class $D$ stochastic processes. We refer the readers to the nice article [4] for related results.

The rest of the paper is organized as follows. In Section 2, we set up the precise framework. Section 3 is devoted to establishing the existence and uniqueness of solutions of a backward stochastic differential equation with singular coefficients. The boundary value problem is solved in Section 4.

\section{Framework}

We assume that $d \geq 3$. Hereafter we fix $p>\frac{d}{2}$. We will use $\langle\cdot, \cdot\rangle$ and $(\cdot, \cdot)$ to denote respectively the inner product of the Euclidean space $\mathbb{R}^{d}$ and the inner product of the $L^{2}(D)$-space. $\|\cdot\|_{\infty}$ denotes the $L^{\infty}$ normal. Recall that $W^{1,2}(D)$ is the Sobolev space on D with the norm $\|u\|_{W^{1,2}(D)}=\left(\int_{D}\langle\nabla u, \nabla u\rangle d x+(u, u)\right)^{\frac{1}{2}}$ and that $W_{0}^{1,2}(D)$ denotes the completion of $C_{0}^{\infty}(D)$ under the same norm $\|\cdot\|_{W^{1,2}(D)}$. The operator $\mathcal{A}$ introduced in Section 1 is rigorously determined by the quadratic form $(\mathcal{Q}, D(\mathcal{Q}))$ with $D(\mathcal{Q})=W^{1,2}(D)$ and for $u, v \in W^{1,2}(D)$

$$
\begin{aligned}
\mathcal{Q}(u, v)= & (-\mathcal{A} u, v)_{L^{2}\left(\mathbb{R}^{d}\right)} \\
= & \frac{1}{2} \sum_{i, j=1}^{d} \int_{\mathbb{R}^{d}} a_{i j}(x) \frac{\partial u}{\partial x_{i}} \frac{\partial v}{\partial x_{j}} d x-\sum_{i=1}^{d} \int_{\mathbb{R}^{d}} b_{i}(x) \frac{\partial u}{\partial x_{i}} v(x) d x \\
& -\sum_{i=1}^{d} \int_{\mathbb{R}^{d}} \hat{b}_{i}(x) \frac{\partial v}{\partial x_{i}} u(x) d x-\int_{D} q(x) u(x) v(x) d x .
\end{aligned}
$$

Definition 2.1 $u \in W^{1,2}(D) \bigcap C(\bar{D})$ is called a weak solution of problem (1.1), if $u$ satisfies

$$
\mathcal{Q}(u, \phi)=\int_{D} f(x, u(x)) \phi(x) d x \text { for any } \phi \in C_{0}^{\infty}(D),
$$

and $u(x)=\varphi(x)$ when $x \in \partial D$.

Now we need to recall the notion of VMO functions in order to apply the $W^{1, p}$ estimates for the divergence operators. A locally integrable function $g$ is said to be in the $V M O$ space, if

$$
\lim _{\varrho \rightarrow 0} \sup _{r \leq \varrho} \sup _{B_{r} \subset D} \frac{1}{\left|B_{r}\right|} \int_{B_{r}}\left|g(x)-g_{B_{r}}\right| d x=0,
$$

where $B_{r}$ denotes a ball of radius $r$ and

$$
g_{B_{r}}=\frac{1}{\left|B_{r}\right|} \int_{B_{r}} g(x) d x .
$$

\section{Assumption I}

(I.1) $\quad D$ is a bounded, connected $C^{1}$-domain.

(I.2) Each $a_{i j}, 1 \leq i, j \leq d$, is continuous and belongs to the $V M O$ space. 
Remark 2.1 Under the Assumption I, it follows from Theorem 1 in [3] that there exists a unique element $v \in W_{0}^{1,2 p}(D)$ such that

$$
\int_{D}\langle a(x) \nabla v(x), \nabla \phi(x)\rangle d x=-2 \int_{D}\langle\hat{b}(x), \nabla \phi(x)\rangle d x, \quad \forall \phi \in W_{0}^{1,2}(D) .
$$

Set $L_{0} u=\frac{1}{2} \sum_{i, j=1}^{d} \frac{\partial}{\partial x_{i}}\left(a_{i j}(x) \frac{\partial u}{\partial x_{j}}\right)$. Let $\left(X_{t}, P_{x}^{0}, x \in \mathbb{R}^{d}\right)$ be the diffusion process generated by the infinitesimal operator $L_{0}$. It is well-known that $\left(X_{t}, P_{x}^{0}\right)$ is a conservative Feller process on $\mathbb{R}^{d}$ that has continuous transition density function which admits a twosided Aronsons heat kernel estimate (see Aronson [1, 2]). Moreover, by Theorem IV.2.5 in [14], for quasi-everywhere (q.e.) $x \in \mathbb{R}^{d}$ we have the Fukushima's decomposition

$$
X(t)-X(0)=M_{t}^{0}+N_{t}^{0}, \quad P_{x}^{0}-a . s .
$$

where $M_{t}^{0}$ is a martingale additive functional (MAF) and $N_{t}^{0}$ is a continuous additive functional (CAF) of locally zero energy. However the decomposition (2.1) can be strengthened to hold for every $x \in \mathbb{R}^{d}$. This follows from the existence of the heat kernel, Theorem 3.5.4 in [15] and Theorem 2 in [11]. Let $v$ be the function stated in Remark 2.1. By Sobolev embedding theorem, $v \in C\left(\mathbb{R}^{d}\right)$ if we extend $v=0$ on $D^{c}$. Moreover, for any $x \in \mathbb{R}^{d}$ the Fukushima's decomposition holds

$$
v(X(t))-v(X(0))=M_{t}^{v}+N_{t}^{v}, \quad P_{x}^{0} \text {-a.e. },
$$

where $M_{t}^{v}$ is a MAF and $N_{t}^{v}$ is a CAF of zero energy.

Assume that $\mathrm{f}(\mathrm{x}, \mathrm{y})$ is a measurable function, which is continuous w.r.t. $y$ and satisfies

$$
\left\{\begin{array}{l}
\left(y_{1}-y_{2}\right)\left(f\left(x, y_{1}\right)-f\left(x, y_{2}\right)\right) \leq-J(x)\left|y_{1}-y_{2}\right|^{2}, \\
|f(x, y)| \leq C\left(1+J_{1}(x)|y|\right),
\end{array}\right.
$$

for some constant $C$ and $J, J_{1} \in L^{p}(D)$. Finally set

$$
\widetilde{a}=\left\{\widetilde{a}_{i j}(x)\right\}_{1 \leq i, j \leq d}=\left\{\frac{a_{i j}(x)+a_{j i}(x)}{2}\right\}_{1 \leq i, j \leq d} .
$$

\section{BSDEs with Singular Coefficients}

In this section, we will obtain the existence and uniqueness of solutions of a class of BSDEs with singular coefficients and random terminal time. The results is of independent interest on its own right and will be used in the subsequent sections. Recall that $X=$ $\left(\Omega, \mathcal{F}, \mathcal{F}_{t}, X_{t}, P_{x}, x \in \mathbb{R}^{d}\right)$ denotes the diffusion process associated with the generator $L_{1}$ given by

$$
L_{1} u=\frac{1}{2} \sum_{i, j=1}^{d} \frac{\partial}{\partial x_{i}}\left(a_{i j}(x) \frac{\partial u}{\partial x_{j}}\right)+\sum_{i=1}^{d} b_{i}(x) \frac{\partial u}{\partial x_{i}},
$$

where $\mathcal{F}_{t}$ is the completed minimal admissible filtration generated by $X_{t}$. Similar to (2.1) we have the following Fukushima's decomposition: for any $x \in \mathbb{R}^{d}$

$$
X(t)-X(0)=M_{t}+N_{t}, \quad P_{x}-\text { a.e. },
$$


where $M_{t}=\left(M_{t}^{1}, M_{t}^{2}, \cdots M_{t}^{d}\right)$ is a MAF of finite energy and $N_{t}$ is CAF of locally zero quadratic variation. Moreover,

$$
\left\langle M^{i}, M^{j}\right\rangle_{t}=\int_{0}^{t} \tilde{a}_{i j}(X(s)) d s .
$$

Given a $h \in L^{p}(D)$. Let $F$ be a Borel measurable function on D satisfying

$$
|F(x)| \leq C(1+|h(x)|),
$$

for some constant $C>0$. Then similar to Theorem 3.18 and Theorem 4.6 in [9], we have the following result.

Lemma 3.1 Assume there exists a $x_{0} \in D$ such that

$$
E_{x_{0}}\left[e^{-\int_{0}^{\tau} D h(X(s)) d s}\right]<\infty,
$$

then for any $F$ satisfying (3.3) we have

$$
\sup _{x \in D} E_{X}\left[\int_{0}^{\tau_{D}} e^{-\int_{0}^{t} h(X(s)) d s} F(X(t)) d t\right]<\infty .
$$

Proof Let $G_{D}(x, y)$ denote the Green function on the domain $D$ associated with the operator $L_{1}$. It is known from (2.14) in [5] that

$$
\int_{D} G_{D}(x, y)|h(y)| d y=E_{x}\left[\int_{0}^{\tau_{D}}|h(X(s))| d s\right] \leq \sigma\|h\|_{L^{p}(D)},
$$

for some constant $\sigma$. Since $h$ is $L^{p}$ integrable, one can choose $\delta>0$ such that for any $B \subset D$ with $m(B)<\delta$, we have $\left\|I_{B} h\right\|_{L^{p}(D)}<\frac{1}{\sigma}$. Hence

$$
\sup _{B \subset D: m(B)<\delta} \sup _{x \in D} \int_{B} G_{D}(x, y)|h(y)| d y<1 .
$$

So $h$ belongs to the class of functions $K_{1}$ defined in [6]. It thus follows from Theorem 2.2 in [6] and (3.4) that

$$
E_{x}\left[e^{-\int_{0}^{\tau} D} h(X(s)) d s\right] \leq C_{1}, \quad \forall x \in D,
$$

where $C_{1}$ is a positive constant. Moreover by (3.6)

$$
\inf _{x \in D} E_{x}\left[e^{-\int_{0}^{\tau} D} h(X(s)) d s\right] \geq \inf _{x \in D} \exp \left(-E_{x}\left[\int_{0}^{\tau_{D}} h(X(s)) d s\right]\right) \geq \exp \left(-\sigma\|h\|_{L^{p}(D)}\right) .
$$

On the other hand, it was shown in $[1,2]$ that the transition density function $p(t, x, y)$ of $\left(X_{t}, P_{x}, x \in \mathbb{R}^{d}\right)$ has a Gaussian upper bound estimate:

$$
p(t, x, y) \leq \frac{\sigma_{1}}{t^{d / 2}} e^{-\frac{\sigma_{2}|x-y|^{2}}{t}}, \quad \forall(t, x, y) \in(0, T] \times \mathbb{R}^{d} \times \mathbb{R}^{d}
$$

for some constants $\sigma_{1}, \sigma_{2}>0$. This bound together with $h \in L^{p}(D)$ implies that

$\sup _{x \in D} E_{x}\left[\int_{0}^{\delta}|h(X(s))| d s\right]<1$ for some $\delta>0$. Hence similar to the proof of Theorem 4.6 in [9] we can show that

$$
\int_{0}^{\infty} \sup _{x \in D} E_{x}\left[e^{-\int_{0}^{t} h(X(s)) d s} ; t<\tau_{D}\right] d t<\infty .
$$

The remainder of the proof of (3.5) is the same as that of Theorem 3.18 in [9]. we omit the details. 
Fix a probability measure $\mu$ on $\mathbb{R}^{d}$ and let $P=P_{\mu}$ denote the probability law of the diffusion process $X$ starting with the initial distribution $\mu$, namely, $P(\cdot)=\int_{\mathbb{R}^{d}} P_{x}(\cdot) \mu(d x)$. We now consider BSDEs driven by the martingale part $M$ in (3.2) with random terminal time on the probability space $\left(\Omega, \mathcal{F}, \mathcal{F}_{t}, P\right)$. Denote by $E$ the expectation under $P$.

Let $g(t, y, \omega):[0, \infty] \times \mathbb{R} \times \Omega \rightarrow \mathbb{R}$ be a progressively measurable function. For notational convenience, we omit the random parameter $\omega$. Given a finite stopping time $\tau$ and a random variable $\xi \in \mathcal{F}_{\tau}$. Let us recall the definition of a solution of the BSDEs with random terminal time.

Definition 3.1 A pair $(Y, Z)$ is a solution to the following BSDE

$$
Y(t)=\xi+\int_{t \wedge \tau}^{\tau} g(s, Y(s)) d s-\int_{t \wedge \tau}^{\tau}\left\langle Z(s), d M_{s}\right\rangle,
$$

if $\mathrm{Y}$ is a $\mathbb{R}$-valued progressively measurable process and $\mathrm{Z}$ is a $\mathbb{R}^{d}$-valued predictable process such that:

$$
g(\cdot, Y .) \in L^{1}([0, \tau], d s), \quad Z . \in L^{2}([0, \tau], d s), \quad P \text {-a.s.. }
$$

and (3.10) is valid $P$-a.s. for every $t \geq 0$,

To prove the existence and uniqueness of a solution to the BSDE (3.10), we need the following lemma which can be found in [4].

Lemma 3.2 If $(Y, Z)$ is a solution to the BSDE (3.10), then for any $0 \leq t \leq u \leq \tau$,

$$
|Y(t)| \leq|Y(u)|+\int_{t}^{u} \frac{Y(s)}{|Y(s)|} I_{\{Y(s) \neq 0\}} g(s, Y(s)) d s-\int_{t}^{u}\left\langle\frac{Y(s)}{|Y(s)|} I_{\{Y(s) \neq 0\}} Z(s), d M_{s}\right\rangle .
$$

Suppose that $g$ is continuous with respect to the variable $y$ and satisfies

$$
\left\{\begin{array}{l}
\left(y_{1}-y_{2}\right)\left(g\left(t, y_{1}\right)-g\left(t, y_{2}\right)\right) \leq-K_{1}(t)\left|y_{1}-y_{2}\right|^{2}, \\
|g(t, y)-g(t, 0)| \leq K_{2}(t)+K_{3}(t)|y|,
\end{array}\right.
$$

where $K_{1}(t), K_{2}(t) \geq 0, K_{3}(t) \geq 0$ are progressively measurable stochastic processes.

For any $0<\beta<1$, denote by $\varphi^{\beta}$ the set of the real-valued, adapted, continuous processes $Y(t)$ such that

$$
\|Y\|_{\varphi^{\beta}}=E\left[\sup _{t \geq 0}|Y(t)|^{\beta}\right]<\infty .
$$

If we define $\rho\left(Y_{1}, Y_{2}\right)=\left\|Y_{1}-Y_{2}\right\|_{\varphi^{\beta}}$ as a distance in $\varphi^{\beta}$, then $\varphi^{\beta}$ is complete under this distance. Denote by $M^{\beta}$ the set of $\mathbb{R}^{d}$-valued predictable processes $Z(t)$ such that

$$
\|Z\|_{M^{\beta}}=E\left[\left(\int_{0}^{\infty}|Z(t)|^{2} d t\right)^{\frac{\beta}{2}}\right]<\infty .
$$

Then $M^{\beta}$ is also complete under the distance $\rho^{\prime}\left(Z_{1}, Z_{2}\right)=\left\|Z_{1}-Z_{2}\right\|_{M^{\beta}}$.

Let $\Gamma$ denote the set of the stopping times. We say that a progressively measurable process $Y$ is in Class (D) if the family $\left\{Y(T) I_{\{T<\infty\}}: T \in \Gamma\right\}$ is uniformly integrable. We put

$$
\|Y\|_{1}=\sup \left\{E\left[|Y(T)| I_{\{T<\infty\}}\right]: T \in \Gamma\right\} .
$$

Then it is known that the space of adapted continuous processes which belong to class (D) is complete under $\|\cdot\|_{1}$ norm, see Chapter VI 21 in [10]. Here is the main result of this section. 
Theorem 3.2 Assume

$$
E\left[e^{-\int_{0}^{\tau} K_{1}(s) d s} \xi\right]<\infty,
$$

and

$$
E\left[\int_{0}^{\tau}\left(e^{-\int_{0}^{t} K_{1}(s) d s}\left(K_{2}(t)+|g(t, 0)|\right)+\left|K_{1}(t)\right|+K_{3}(t)\right) d t\right]<\infty .
$$

Then the BSDE (3.10) has a unique solution $(Y, Z)$ such that $e^{-\int_{0}^{t} K_{1}(s) d s} Y(t)$ belongs to Class (D). Moreover, for any $0<\beta<1,\left(e^{-\int_{0}^{t} K_{1}(s) d s} Y(t), e^{-\int_{0}^{t} K_{1}(s) d s} Z(t)\right) \in \varphi^{\beta} \times M^{\beta}$.

Proof We set $g(s, y)=K_{1}(s)=K_{2}(s)=K_{3}(s)=0$ for $s \geq \tau$ for convenience. Set $\tilde{Y}(t)=e^{-\int_{0}^{t} K_{1}(s) d s} Y(t), \tilde{Z}(t)=e^{-\int_{0}^{t} K_{1}(s) d s} Z(t), \tilde{g}(t, y)=e^{-\int_{0}^{t} K_{1}(s) d s} g\left(t, e^{\int_{0}^{t} K_{1}(s) d s} y\right)+$ $K_{1}(t) y$ and $\tilde{\xi}=e^{-\int_{0}^{\tau} K_{1}(s) d s} \xi$. Then it is easy to see that $(Y, Z)$ is a solution to the BSDE (3.10) if and only if $(\tilde{Y}, \tilde{Z})$ satisfies the following BSDE:

$$
\tilde{Y}(t)=\tilde{\xi}+\int_{t \wedge \tau}^{\tau} \tilde{g}(s, \tilde{Y}(s)) d s-\int_{t \wedge \tau}^{\tau}\left\langle\tilde{Z}(s), d M_{s}\right\rangle .
$$

Therefore, we only need to establish the existence and uniqueness of the BSDE (3.16). Note that $\tilde{g}(t, y)$ is also continuous with respect to $y$ and

$$
\left\{\begin{array}{l}
\left(y_{1}-y_{2}\right)\left(\tilde{g}\left(t, y_{1}\right)-\tilde{g}\left(t, y_{2}\right)\right) \leq 0 \\
E\left[\int_{0}^{\tau}|\tilde{g}(t, 0)| d t\right]<\infty
\end{array}\right.
$$

Moreover for any $n \in \mathbb{N}$,

$E\left[\int_{0}^{n} e^{-\int_{0}^{t} K_{1}(s) d s} K_{2}(t) d t\right]=E\left[\int_{0}^{\tau \wedge n} e^{-\int_{0}^{t} K_{1}(s) d s} K_{2}(t) d t\right] \leq E\left[\int_{0}^{\tau} e^{-\int_{0}^{t} K_{1}(s) d s} K_{2}(t) d t\right]<\infty$, where the first inequality is due to the fact $K_{1}(s)=K_{2}(s)=0$ when $s \geq \tau$.

By (3.11) and (3.15), for any $r>0$, we have

$$
\begin{aligned}
& \sup _{|y| \leq r}|\tilde{g}(t, y)-\tilde{g}(t, 0)| \\
\leq & \sup _{|y| \leq r} e^{-\int_{0}^{t} K_{1}(s) d s}\left|g\left(t, e^{\int_{0}^{t} K_{1}(s) d s} y\right)-g(t, 0)\right|+\left|K_{1}(t)\right| r \\
\leq & e^{-\int_{0}^{t} K_{1}(s) d s} K_{2}(t)+\left(\left|K_{1}(t)\right|+K_{3}(t)\right) r \in L^{1}([0, n] \times \Omega), \quad \forall n \in \mathbb{N} .
\end{aligned}
$$

Set $\tilde{\xi}^{N}=(-N) \vee \tilde{\xi} \wedge N$. Note that the martingale representation theorem with respect to the martingale part $M$ is valid according to Theorem 2.1 in [18]. Thus, by (3.17)-(3.18) and Proposition 6.4 in [4] for any $n \in \mathbb{N}$, there exists a solution pair $\left(\tilde{Y_{n}^{N}}, \tilde{Z_{n}^{N}}\right)$ to the following BSDE

$$
\tilde{Y_{n}^{N}}(t)=E\left[\tilde{\xi}^{N} \mid \mathcal{F}_{n}\right]+\int_{t}^{n} I_{s \leq \tau} \tilde{g}\left(s, \tilde{Y_{n}^{N}}(s)\right) d s-\int_{t}^{n}\left\langle\tilde{Z_{n}^{N}}(s), d M_{s}\right\rangle, \quad 0 \leq t \leq n,
$$

and moreover $\tilde{Y_{n}^{N}} \in$ Class $(\mathrm{D}),\left(\tilde{Y_{n}^{N}}, \tilde{Z_{n}^{N}}\right) \in \bigcap_{0<\beta<1} \varphi^{\beta} \times M^{\beta}$, where we set $\tilde{Y_{n}^{N}}(t)=$ $E\left[\tilde{\xi}^{N} \mid \mathcal{F}_{n}\right], \tilde{Z_{n}^{N}}(t)=0$ for $t>n$.

Next we are going to show that $\left\{\tilde{Y_{n}^{N}}\right\}_{n \geq 1}$ is a Cauchy sequence under norm $\|\cdot\|_{1}$.

For $n, i \in \mathbb{N}$, set $\delta \tilde{Y}_{n}^{n+i}(s)=\tilde{Y}_{n+i}^{N}(s)-\tilde{Y_{n}^{N}}(s), \delta \tilde{Z}_{n}^{n+i}(s)=\tilde{Z}_{n+i}^{N}(s)-\tilde{Z_{n}^{N}}(s)$ and $\delta \tilde{g}_{n}(s, y)=I_{s \leq \tau}\left[\tilde{g}\left(s, \tilde{Y_{n}^{N}}(s)+y\right)-\tilde{g}\left(s, \tilde{Y_{n}^{N}}(s)\right)+\tilde{g}\left(s, \tilde{Y_{n}^{N}}(n)\right) I_{s \geq n}\right]$. Then $\left(y_{1}-\right.$ $\left.y_{2}\right)\left(\delta \tilde{g}_{n}\left(t, y_{1}\right)-\delta \tilde{g}\left(t, y_{2}\right)\right) \leq 0$. By (3.19) we have for any $t \geq 0$

$$
\begin{aligned}
\delta \tilde{Y}_{n}^{n+i}(t)= & E\left[\tilde{\xi}^{N} \mid \mathcal{F}_{n+i}\right]-E\left[\tilde{\xi}^{N} \mid \mathcal{F}_{n}\right]+\int_{t \wedge(n+i)}^{n+i} \delta \tilde{g}_{n}\left(s, \delta \tilde{Y}_{n}^{n+i}(s)\right) d s \\
& -\int_{t \wedge(n+i)}^{n+i}\left\langle\delta \tilde{Z}_{n}^{n+i}(s), d M_{s}\right\rangle .
\end{aligned}
$$


Choose a sequence of stopping times $\left\{\tau_{k}\right\}_{k \geq 1}$ that increases to $\infty$ and such that $\int_{0}^{t \wedge \tau_{k}}\left\langle\frac{\delta \tilde{Y}_{n}^{n+i}(s)}{\left|\delta \tilde{Y}_{n}^{n+i}(s)\right|} \delta \tilde{Z}_{n}^{n+i}(s), d M_{s}\right\rangle$ is a martingale. Then by (3.20) we have

$$
\begin{aligned}
\delta \tilde{Y}_{n}^{n+i}\left(t \wedge \tau_{k}\right)= & \delta \tilde{Y}_{n}^{n+i}\left((n+i) \wedge \tau_{k}\right)+\int_{t \wedge(n+i) \wedge \tau_{k}}^{(n+i) \wedge \tau_{k}} \delta \tilde{g}_{n}\left(s, \delta \tilde{Y}_{n}^{n+i}(s)\right) d s \\
& -\int_{t \wedge(n+i) \wedge \tau_{k}}^{(n+i) \wedge \tau_{k}}\left\langle\delta \tilde{Z}_{n}^{n+i}(s), d M_{s}\right\rangle .
\end{aligned}
$$

It follows from Lemma 3.2 that

$$
\begin{aligned}
\left|\delta \tilde{Y}_{n}^{n+i}\left(t \wedge \tau_{k}\right)\right| \leq & \left|\delta \tilde{Y}_{n}^{n+i}\left((n+i) \wedge \tau_{k}\right)\right|+\int_{t \wedge(n+i) \wedge \tau_{k}}^{(n+i) \wedge \tau_{k}} \frac{\delta \tilde{Y}_{n}^{n+i}(s)}{\left|\delta \tilde{Y}_{n}^{n+i}(s)\right|} \delta \tilde{g}_{n}\left(s, \delta \tilde{Y}_{n}^{n+i}(s)\right) d s \\
& -\int_{t \wedge(n+i) \wedge \tau_{k}}^{(n+i) \wedge \tau_{k}}\left\langle\frac{\delta \tilde{Y}_{n}^{n+i}(s)}{\left|\delta \tilde{Y}_{n}^{n+i}(s)\right|} \delta \tilde{Z}_{n}^{n+i}(s), d M_{s}\right\rangle \\
\leq & \left|\delta \tilde{Y}_{n}^{n+i}\left((n+i) \wedge \tau_{k}\right)\right|+\int_{t \wedge(n+i) \wedge \tau_{k}}^{(n+i) \wedge \tau_{k}}\left|\delta \tilde{g}_{n}(s, 0)\right| d s \\
& -\int_{t \wedge(n+i) \wedge \tau_{k}}^{(n+i) \wedge \tau_{k}}\left\langle\frac{\delta \tilde{Y}_{n}^{n+i}(s)}{\left|\delta \tilde{Y}_{n}^{n+i}(s)\right|} \delta \tilde{Z}_{n}^{n+i}(s), d M_{s}\right\rangle .
\end{aligned}
$$

Take the conditional expectation with respect to $\mathcal{F}_{t}$ to obtain

$$
\begin{aligned}
\left|\delta \tilde{Y}_{n}^{n+i}\left(t \wedge \tau_{k}\right)\right| & \leq E\left[\left|\delta \tilde{Y}_{n}^{n+i}\left((n+i) \wedge \tau_{k}\right)\right| \mid \mathcal{F}_{t}\right]+E\left[\int_{t \wedge(n+i) \wedge \tau_{k}}^{(n+i) \wedge \tau_{k}}\left|\delta \tilde{g}_{n}(s, 0)\right| d s \mid \mathcal{F}_{t}\right] \\
& =E\left[\left|\delta \tilde{Y}_{n}^{n+i}\left((n+i) \wedge \tau_{k}\right)\right| \mid \mathcal{F}_{t}\right]+E\left[\int_{t \wedge(n+i) \wedge \tau_{k}}^{(n+i) \wedge \tau_{k}}\left|\delta \tilde{g}_{n}(s, 0)\right| d s \mid \mathcal{F}_{t}\right]
\end{aligned}
$$

here we have used the fact that $\int_{0}^{(n+i) \wedge \tau_{k} \wedge t}\left\langle\frac{\delta \tilde{Y}_{n}^{n+i}(s)}{\left|\delta \tilde{Y}_{n}^{n+i}(s)\right|} \delta \tilde{Z}_{n}^{n+i}(s), d M_{s}\right\rangle, t \geq 0$ is also a martingale with respect to the filtration $\mathcal{F}_{t}, t \geq 0$.

Since $\delta \tilde{Y}_{n}^{n+i}=\tilde{Y}_{n+i}^{N}-\tilde{Y}_{n}^{N}$ is also in Class (D) and $\delta \tilde{Y}_{n}^{n+i}(t)$ is continuous, we get

$$
\lim _{k \rightarrow \infty} \delta \tilde{Y}_{n}^{n+i}\left((n+i) \wedge \tau_{k}\right)=\delta \tilde{Y}_{n}^{n+i}(n+i) \text { in } L^{1}(P ; \Omega) .
$$

Since

$$
\begin{aligned}
& \int_{t \wedge(n+i) \wedge \tau_{k}}^{(n+i) \wedge \tau_{k}}\left|\delta \tilde{g}_{n}(s, 0)\right| d s \\
\leq & \int_{n}^{(n+i) \wedge \tau}\left(\left|\tilde{g}\left(s, \tilde{Y_{n}^{N}}(n)\right)-\tilde{g}(s, 0)\right|+|\tilde{g}(s, 0)|\right) d s \\
\leq & \int_{n}^{(n+i) \wedge \tau}\left[K_{2}(s) e^{-\int_{0}^{s} K_{1}(r) d r}+\left(\left|K_{1}(s)\right|+K_{3}(s)\right)\left|\tilde{Y_{n}^{N}}(n)\right|\right] d s+\int_{n}^{(n+i) \wedge \tau}(|\tilde{g}(s, 0)|) d s \\
\leq & \int_{n}^{(n+i) \wedge \tau} K_{2}(s) e^{-\int_{0}^{s} K_{1}(r) d r} d s+N \int_{n}^{(n+i) \wedge \tau}\left(\left|K_{1}(s)\right|+K_{3}(s)\right) d s \\
& +\int_{n}^{(n+i) \wedge \tau}(|\tilde{g}(s, 0)|) d s \in L^{1}(P ; \Omega),
\end{aligned}
$$

by the dominated convergence theorem we have

$$
\lim _{k \rightarrow \infty} \int_{t \wedge \tau_{k}}^{(n+i) \wedge \tau_{k}}\left|\delta \tilde{g}_{n}(s, 0)\right| d s=\int_{t \wedge \tau}^{(n+i) \wedge \tau}\left|\delta \tilde{g}_{n}(s, 0)\right| d s \text { in } L^{1}(P ; \Omega) .
$$

Letting $k \rightarrow \infty$ in (3.21) and noticing $\delta \tilde{g}_{n}(s, 0)=0$ for $s>\tau$, it follows from (3.22) and (3.24) that

$$
\left|\delta \tilde{Y}_{n}^{n+i}(t)\right| \leq E\left[\left|\delta \tilde{Y}_{n}^{n+i}(n+i)\right| \mid \mathcal{F}_{t}\right]+E\left[\int_{n \wedge \tau}^{(n+i) \wedge \tau}\left|\delta \tilde{g}_{n}(s, 0)\right| d s \mid \mathcal{F}_{t}\right] .
$$

Hence,

$$
\begin{aligned}
\left\|\delta \tilde{Y}_{n}^{n+i}\right\|_{1} & =\sup _{T: T \in \Gamma} E\left[\left|\delta \tilde{Y}_{n}^{n+i}(T)\right| I_{\{T<\infty\}}\right] \\
& \leq \sup _{T: T \in \Gamma} E\left[E\left[\mid \delta \tilde{Y}_{n}^{n+i}(n+i) \| \mathcal{F}_{T}\right]\right]+E\left[E\left[\int_{n \wedge \tau}^{(n+i) \wedge \tau}\left|\delta \tilde{g}_{n}(s, 0)\right| d s \mid \mathcal{F}_{T}\right]\right] \\
& =E\left[\left|\delta \tilde{Y}_{n}^{n+i}(n+i)\right|\right]+E\left[\int_{n \wedge \tau}^{(n+i) \wedge \tau}\left|\tilde{g}\left(s, \tilde{Y}_{n}^{N}(n)\right)\right| d s\right] .
\end{aligned}
$$

Since $\tilde{\xi}^{N}$ is bounded, it holds that

$$
\delta \tilde{Y}_{n}^{n+i}(n+i)=E\left[\tilde{\xi}^{N} \mid \mathcal{F}_{n+i}\right]-E\left[\tilde{\xi}^{N} \mid \mathcal{F}_{n}\right] \rightarrow 0 \text { in } L^{1}(\Omega)
$$


as $n \rightarrow \infty$. By (3.11), we have

$$
\begin{aligned}
& \left|\tilde{g}\left(s, \tilde{Y_{n}^{N}}(n)\right)\right| \\
\leq & e^{-\int_{0}^{s} K_{1}(r) d r}\left(\left|g\left(s, e^{\int_{0}^{s} K_{1}(r) d r} \tilde{Y_{n}^{N}}(n)\right)-g(s, 0)\right|\right) \\
& +e^{-\int_{0}^{s} K_{1}(r) d r}|g(s, 0)|+N\left|K_{1}(s)\right| \\
\leq & e^{-\int_{0}^{s} K_{1}(r) d r} K_{2}(s)+N K_{3}(s) \\
& +e^{-\int_{0}^{s} K_{1}(r) d r}|g(s, 0)|+N\left|K_{1}(s)\right| .
\end{aligned}
$$

Thus the condition (3.15) implies that

$$
E\left[\int_{0}^{\tau}\left|\tilde{g}\left(s, \tilde{Y_{n}^{N}}(n)\right)\right| d s\right]<\infty .
$$

Combining (3.26), (3.27) and (3.29) we see that $\left\{\tilde{Y_{n}^{N}}\right\}_{n \geq 1}$ is a Cauchy sequence with respect to the $\|\cdot\|_{1}$ norm. Thus there exists a $\tilde{Y}^{N} \in$ Class (D) such that $\lim _{n \rightarrow \infty} \| \tilde{Y_{n}^{N}}-$ $\tilde{Y}^{N} \|_{1}=0$. It follows from [10] that there exists a subsequence of $\left\{\tilde{Y_{n}^{N}}\right\}_{n \geq 1}$ that converges almost surely to $\tilde{Y}^{N}$ uniformly on $[0, \infty)$. Hence $\tilde{Y}^{N}$ is a continuous process.

Next we will show that $\tilde{Y_{n}^{N}}$ also converges to $\tilde{Y}^{N}$ in $\varphi^{\beta}$ for any $0<\beta<1$.

Let $\eta_{n}=\left|\delta \tilde{Y}_{n}^{n+i}(n+i)\right|+\int_{n \wedge \tau}^{(n+i) \wedge \tau}\left|\delta \tilde{g}_{n}(s, 0)\right| d s, M_{n}(t)=E\left[\eta_{n} \mid \mathcal{F}_{t}\right]$. Then by Lemma 6.1 in [4] we have $E\left[\sup _{t \geq 0}\left|M_{n}(t)\right|^{\beta}\right] \leq \frac{1}{1-\beta} E\left[\left|\eta_{n}\right|\right]^{\beta}$. Together with (3.25) we get

$$
E\left[\sup _{t \geq 0}\left|\delta \tilde{Y}_{n}^{n+i}(t)\right|^{\beta}\right] \leq E\left[\sup _{t \geq 0}\left|M_{n}(t)\right|^{\beta}\right] \leq \frac{1}{1-\beta} E\left[\left|\eta_{n}\right|\right]^{\beta} \rightarrow 0
$$

as $n \rightarrow 0$. Therefor $\tilde{Y}^{N} \in \varphi^{\beta}$ and

$$
\lim _{n \rightarrow \infty} E\left[\sup _{t \geq 0}\left|\tilde{Y_{n}^{N}}(t)-\tilde{Y}^{N}(t)\right|^{\beta}\right]=0 .
$$

Next we will establish the convergence of $\left\{\tilde{Z_{n}^{N}}\right\}_{n \geq 1}$.

By (3.20) and Ito's formula,

$$
\begin{aligned}
& \left|\delta \tilde{Y}_{n}^{n+i}(t)\right|^{2}+\int_{t}^{n+i}\left\langle\tilde{a}(X(s)) \delta \tilde{Z}_{n}^{n+i}(s), \delta \tilde{Z}_{n}^{n+i}(s)\right\rangle d s \\
= & \left|\delta \tilde{Y}_{n}^{n+i}(n+i)\right|^{2}+2 \int_{t}^{(n+i)} \delta \tilde{Y}_{n}^{n+i}(s) \delta \tilde{g}_{n}\left(s, \delta \tilde{Y}_{n}^{n+i}(s)\right) d s \\
& -2 \int_{t}^{n}\left\langle\delta \tilde{Y}_{n}^{n+i}(s) \delta \tilde{Z}_{n}^{n+i}(s), d M_{s}\right\rangle .
\end{aligned}
$$

Using (1.2) and (3.17), we deduce that

$$
\begin{aligned}
\lambda \int_{0}^{n+i}\left|\delta \tilde{Z}_{n}^{n+i}(s)\right|^{2} d s \leq & \left|\delta \tilde{Y}_{n}^{n+i}(n+i)\right|^{2}+2 \int_{0}^{(n+i)} \delta \tilde{Y}_{n}^{n+i}(s) \delta \tilde{g}_{n}(s, 0) d s \\
& -2 \int_{0}^{n+i}\left\langle\delta \tilde{Y}_{n}^{n+i}(s) \delta \tilde{Z}_{n}^{n+i}(s), d M_{s}\right\rangle .
\end{aligned}
$$

It follows now from the Burkholder's inequality that

$$
\begin{aligned}
& E\left[\left(\int_{0}^{\infty}\left|\delta \tilde{Z}_{n}^{n+i}(s)\right|^{2} d s\right)^{\frac{\beta}{2}}\right]=E\left[\left(\int_{0}^{n+i}\left|\delta \tilde{Z}_{n}^{n+i}(s)\right|^{2} d s\right)^{\frac{\beta}{2}}\right] \\
\leq & C_{\beta} E\left[\left|\delta \tilde{Y}_{n}^{n+i}(n+i)\right|^{\beta}\right]+2 E\left[\sup _{t \geq 0}\left|\delta \tilde{Y}_{n}^{n+i}(t)\right|^{\frac{\beta}{2}}\left(\int_{n}^{(n+i)} \delta \tilde{g}_{n}(s, 0) d s\right)^{\frac{\beta}{2}}\right] \\
& +2 C_{\beta} E\left[\left(\int_{0}^{n+i}\left|\delta \tilde{Y}_{n}^{n+i}(s)\right|^{2}\left|\delta \tilde{Z}_{n}^{n+i}(s)\right|^{2} d s\right)^{\frac{\beta}{4}}\right] \\
\leq & C_{\beta}\left\{E\left[\left|\delta \tilde{Y}_{n}^{n+i}(n+i)\right|^{\beta}\right]+E\left[\sup _{t \geq 0}\left|\delta \tilde{Y}_{n}^{n+i}(t)\right|^{\beta}\right]+E\left[\left(\int_{n}^{(n+i)} \delta \tilde{g}_{n}(s, 0) d s\right)^{\beta}\right]\right\} \\
& +2 C_{\beta} E\left[\sup _{t \geq 0}\left|\delta \tilde{Y}_{n}^{n+i}(t)\right|^{\frac{\beta}{2}}\left(\int_{0}^{n+i}\left|\delta \tilde{Z}_{n}^{n+i}(s)\right|^{2} d s\right)^{\frac{\beta}{4}}\right] \\
\leq & C_{\beta}\left\{2 \sup _{t \geq 0} E\left[\left|\delta \tilde{Y}_{n}^{n+i}(t)\right|^{\beta}\right]+\left[E\left(\int_{n}^{(n+i)} \delta \tilde{g}_{n}(s, 0) d s\right)\right]^{\beta}\right\} \\
& +2 C_{\beta}^{2} E\left[\sup _{t \geq 0}\left|\delta \tilde{Y}_{n}^{n+i}(t)\right|^{\beta}\right]+\frac{1}{2} E\left[\left(\int_{0}^{n+i}\left|\delta \tilde{Z}_{n}^{n+i}(s)\right|^{2} d s\right)^{\frac{\beta}{2}}\right] .
\end{aligned}
$$


Thus

$E\left[\left(\int_{0}^{\infty}\left|\delta \tilde{Z}_{n}^{n+i}(s)\right|^{2} d s\right)^{\beta}\right] \leq\left(4 C_{\beta}+4 C_{\beta}^{2}\right) E\left[\sup _{t \geq 0}\left|\delta \tilde{Y}_{n}^{n+i}(t)\right|^{\beta}\right]+2 C_{\beta}\left[E\left(\int_{n}^{(n+i)} \delta \tilde{g}_{n}(s, 0) d s\right)\right]^{\beta} \rightarrow 0$ as $n \rightarrow \infty$, where (3.17) was used. So there exists $\tilde{Z}^{N} \in M^{\beta}$ such that $\lim _{n \rightarrow \infty} E\left[\left(\int_{0}^{\infty}\left|\tilde{Z}_{n}^{n+i}(s)-\tilde{Z}^{N}(s)\right|^{2} d s\right)^{\frac{\beta}{2}}\right]=0$.

Now we will show that $\left(\tilde{Y}^{N}, \tilde{Z}^{N}\right)$ is a solution of the equation

$$
\tilde{Y}^{N}(t)=\tilde{\xi}^{N}+\int_{t \wedge \tau}^{\tau} \tilde{g}\left(s, \tilde{Y}^{N}(s)\right) d s-\int_{t \wedge \tau}^{\tau}\left\langle\tilde{Z}^{N}(s), d M_{s}\right\rangle .
$$

By (3.30) we can find a subsequence $\left\{n_{k}\right\}_{k \geq 1}$ such that

$$
\lim _{n_{k} \rightarrow \infty} \sup _{s \geq 0}\left|\tilde{Y}_{n_{k}}^{N}(s)-\tilde{Y}^{N}(s)\right|=0, \quad P \text {-a.e.. }
$$

By (3.17) and (3.18) for P-a.e. $\omega$,

$$
\begin{aligned}
\left|\tilde{g}\left(s, \tilde{Y}_{n_{k}}^{N}(s)\right)\right| & \leq|\tilde{g}(s, 0)|+K_{2}(s) e^{-\int_{0}^{s} K_{1}(r) d r}+\left|K_{1}(s) \tilde{Y}_{n_{k}}^{N}(s)\right|+\left|K_{3}(s) \tilde{Y}_{n_{k}}^{N}(s)\right| \\
& \leq|\tilde{g}(s, 0)|+K_{2}(s) e^{-\int_{0}^{s} K_{1}(r) d r}+C(\omega)\left(\left|K_{1}(s)\right|+K_{3}(s)\right) \in L^{1}([0, \tau(\omega)], d s),
\end{aligned}
$$

where $C(\omega)=\sup _{s \geq 0} \sup _{n_{k}: k \geq 1}\left|\tilde{Y}_{n_{k}}^{N}(s, \omega)\right|+\left|\tilde{Y}^{N}(s, \omega)\right|$. Hence using the dominate convergence theorems we get

$$
\lim _{n_{k} \rightarrow \infty} \int_{t \wedge \tau}^{\tau} \tilde{g}\left(s, \tilde{Y}_{n_{k}}^{N}(s)\right) d s=\int_{t \wedge \tau}^{\tau} \tilde{g}\left(s, \tilde{Y}^{N}(s)\right) d s, \quad P-a . s . .
$$

On the other hand, since $\tilde{Z_{n}^{N}}$ converges to $\tilde{Z}^{N}$ in $M^{\beta}, \int_{t \wedge \tau}^{\tau}\left\langle\tilde{Z_{n}^{N}}(s), d M_{s}\right\rangle$ converges to $\int_{t \wedge \tau}^{\tau}\left\langle\tilde{Z}^{N}(s), d M_{s}\right\rangle$ in probability as $n \rightarrow \infty$. Note also that $E\left[\tilde{\xi}^{N} \mid \mathcal{F}_{n_{k}}\right]$ converges to $\tilde{\xi}^{N}$ in probability as $k \rightarrow \infty$. So replacing $n$ by $n_{k}$ in (3.19) and letting $k \rightarrow \infty$ we see that $\left(\tilde{Y}^{N}, \tilde{Z}^{N}\right)$ satisfies (3.31).

Finally we show that there exists a solution to $\operatorname{BSDE}$ (3.16). For $N, N_{1} \in \mathbb{N}$, let $\delta \tilde{Y}^{N}(t)=\tilde{Y}^{N+N_{1}}(t)-\tilde{Y}^{N}(t), \delta \tilde{Z}^{N}(t)=\tilde{Z}^{N+N_{1}}(t)-\tilde{Z}^{N}(t)$ and $\delta \tilde{g}(t, y)=\tilde{g}(t, y+$ $\left.\tilde{Y}^{N}(t)\right)-\tilde{g}\left(t, \tilde{Y}^{N}(t)\right)$. Then by (3.17)

$$
y \cdot \delta \tilde{g}(t, y)=y\left(\tilde{g}\left(t, y+\tilde{Y}^{N}(t)\right)-\tilde{g}\left(t, \tilde{Y}^{N}(t)\right)\right) \leq 0 .
$$

From the equation (3.31) we have

$$
\delta \tilde{Y}^{N}(t)=\tilde{\xi}^{N+N_{1}}-\tilde{\xi}^{N}+\int_{t \wedge \tau}^{\tau} \delta \tilde{g}\left(s, \delta \tilde{Y}^{N}(s)\right) d s-\int_{t \wedge \tau}^{\tau}\left\langle\delta \tilde{Z}^{N}(s), d M_{s}\right\rangle .
$$

Since $\delta \tilde{Y}^{N}(t)=\tilde{Y}^{N+N_{1}}(t)-\tilde{Y}^{N}(t)$ is also in Class (D), using Lemma 2.2 and the similar arguments as in the proof of (3.25), we obtain

$$
\left|\delta \tilde{Y}^{N}(t)\right| \leq E\left[\mid \tilde{\xi}^{N+N_{1}}-\tilde{\xi}^{N} \| \mathcal{F}_{t}\right]
$$

This together with (3.14) imply

$$
\left\|\delta \tilde{Y}^{N}\right\|_{1}=\sup _{T: T \in \Gamma} E\left[\left|\delta \tilde{Y}^{N}(T)\right| I_{\{T<\infty\}}\right] \leq E\left[\left|\tilde{\xi}^{N+N_{1}}-\tilde{\xi}^{N}\right|\right] \rightarrow 0
$$

as $N \rightarrow \infty$. Hence there exists a continuous process $\tilde{Y} \in$ Class (D) such that $\lim _{N \rightarrow \infty}\left\|\tilde{Y}^{N}-\tilde{Y}\right\|_{1}=0$. For any $0<\beta<1$, using Lemma 6.1 in [4] and (3.34) we get

$$
E\left[\sup _{t \geq 0}\left|\delta \tilde{Y}^{N}(t)\right|^{\beta}\right] \leq \frac{1}{1-\beta}\left(E\left|\tilde{\xi}^{N+N_{1}}-\tilde{\xi}^{N}\right|\right)^{\beta} .
$$


This implies that

$$
\lim _{N \rightarrow \infty} E\left[\sup _{t \geq 0}\left|\tilde{Y}^{N}(t)-\tilde{Y}(t)\right|^{\beta}\right]=0 .
$$

Applying Ito's formula to $\tilde{Y}^{N}$ in (3.33) and using (3.32), we obtain

$$
\begin{aligned}
& \left|\delta \tilde{Y}^{N}(t)\right|^{2}+\int_{t \wedge \tau}^{\tau}\left\langle\tilde{a}(X(s)) \delta \tilde{Z}^{N}(s), \delta \tilde{Z}^{N}(s)\right\rangle d s \\
\leq & \left|\tilde{\xi}^{N+N_{1}}-\tilde{\xi}^{N}\right|^{2}+2 \int_{t \wedge \tau}^{\tau} \delta \tilde{Y}^{N}(s) \delta \tilde{g}\left(s, \delta \tilde{Y}^{N}(s)\right) d s \\
& -2 \int_{t \wedge \tau}^{\tau}\left\langle\delta \tilde{Y}^{N}(s) \delta \tilde{Z}^{N}(s), d M_{s}\right\rangle \\
\leq & \left|\tilde{\xi}^{N+N_{1}}-\tilde{\xi}^{N}\right|^{2}-2 \int_{t \wedge \tau}^{\tau}\left\langle\delta \tilde{Y}^{N}(s) \delta \tilde{Z}^{N}(s), d M_{s}\right\rangle .
\end{aligned}
$$

By (1.2), (3.37) and Burkholder's inequality, we have

$$
\begin{aligned}
E\left[\left(\int_{0}^{\tau}\left|\delta \tilde{Z}^{N}(s)\right|^{2} d s\right)^{\frac{\beta}{2}}\right] & \leq C_{\beta}\left\{E\left[|\tilde{\xi}|_{|\tilde{\xi}| \geq N}^{\beta}\right]+2 C_{\beta} E\left[\left(\int_{0}^{\tau}\left|\delta \tilde{Y}^{N}(s) \delta \tilde{Z}^{N}(s)\right|^{2} d s\right)^{\frac{\beta}{4}}\right]\right\} \\
& \leq C_{\beta}\left\{\left(E\left[|\tilde{\xi}|_{|\tilde{\xi}| \geq N}\right]\right)^{\beta}+2 E\left[\sup _{s \geq 0}\left|\delta \tilde{Y}^{N}(s)\right|^{\frac{\beta}{2}}\left(\int_{0}^{\tau}\left|\delta \tilde{Z}^{N}(s)\right|^{2} d s\right)^{\frac{\beta}{4}}\right]\right\} \\
& \leq C_{\beta}\left(E\left[|\tilde{\xi}|_{|\tilde{\xi}| \geq N}\right]\right)^{\beta}+2 C_{\beta}^{2} E\left[\sup _{s \geq 0}\left|\delta \tilde{Y}^{N}(s)\right|^{\beta}\right]+\frac{1}{2} E\left[\left(\int_{0}^{\tau}\left|\delta \tilde{Z}^{N}(s)\right|^{2} d s\right)^{\frac{\beta}{2}}\right] .
\end{aligned}
$$

(3.38), (3.14) and (3.35)imply that

$$
E\left[\left(\int_{0}^{\tau}\left|\delta \tilde{Z}^{N}(s)\right|^{2} d s\right)^{\frac{\beta}{2}}\right] \leq 2 C_{\beta}\left(E\left[|\tilde{\xi}|_{|\tilde{\xi}| \geq N}\right]\right)^{\beta}+4 C_{\beta}^{2} E\left[\sup _{s \geq 0}\left|\delta \tilde{Y}^{N}(s)\right|^{\beta}\right] \rightarrow 0
$$

as $N \rightarrow \infty$. So there exists $\tilde{Z} \in M^{\beta}$ such that

$$
\lim _{N \rightarrow \infty} E\left[\left(\int_{0}^{\infty}\left|\tilde{Z}^{N}(s)-\tilde{Z}(s)\right|^{2} d s\right)^{\frac{\beta}{2}}\right]=\lim _{N \rightarrow \infty} E\left[\left(\int_{0}^{\tau}\left|\tilde{Z}^{N}(s)-\tilde{Z}(s)\right|^{2} d s\right)^{\frac{\beta}{2}}\right]=0 .
$$

Letting $N \rightarrow \infty$ in (3.31), it is easy to see that $(\tilde{Y}, \tilde{Z})$ is a solution to the BSDE (3.16).

Now we proceed to prove the uniqueness of the BSDE (3.16).

Suppose $\left(\tilde{Y}_{1}, \tilde{Z}_{1}\right)$ is another solution to (3.16) such that $\tilde{Y}_{1} \in$ Class (D). Let $\delta \tilde{Y}(t)=$ $\tilde{Y}(t)-\tilde{Y}_{1}(t), \delta \tilde{Z}(t)=\tilde{Z}(t)-\tilde{Z}_{1}(t)$. Choose a sequence of stopping times $\left\{T_{k}\right\}_{k \geq 1}$ that increases to $\infty$ and such that $\int_{0}^{t \wedge T_{k}}\left\langle\frac{\delta \tilde{Y}(s)}{|\delta \tilde{Y}(s)|} \delta \tilde{Z}(s), d M_{s}\right\rangle$ is a uniformly integrable martingale for every $k$. Then by Lemma 3.2 we have

$$
\begin{aligned}
\left|\delta \tilde{Y}\left(t \wedge T_{k}\right)\right| \leq & \left|\delta \tilde{Y}\left(\tau \wedge T_{k}\right)\right|+\int_{t \wedge T_{k}}^{\tau \wedge T_{k}} \frac{\delta \tilde{Y}(s)}{|\delta \tilde{Y}(s)|}\left(\tilde{g}\left(s, \tilde{Y}_{1}(s)\right)-\tilde{g}\left(s, \tilde{Y}_{2}(s)\right)\right) d s \\
& -\int_{t \wedge T_{k}}^{\tau \wedge T_{k}}\left\langle\frac{\delta \tilde{Y}(s)}{|\delta \tilde{Y}(s)|} \delta \tilde{Z}(s), d M_{s}\right\rangle \\
\leq & \left|\delta \tilde{Y}\left(\tau \wedge T_{k}\right)\right|-\int_{t \wedge T_{k}}^{\tau \wedge T_{k}}\left\langle\frac{\delta \tilde{Y}(s)}{|\delta \tilde{Y}(s)|} \delta \tilde{Z}(s), d M_{s}\right\rangle .
\end{aligned}
$$

Taking the conditional expectation with respect to $\mathcal{F}_{t}$ on both sides, we obtain

$$
\left|\delta \tilde{Y}\left(t \wedge T_{k}\right)\right| \leq E\left[\left|\delta \tilde{Y}\left(\tau \wedge T_{k}\right)\right| \mid \mathcal{F}_{t}\right] .
$$

Since $\delta \tilde{Y}(t)=\tilde{Y}(t)-\tilde{Y}_{1}(t)$ is in Class (D) and $\lim _{k \rightarrow \infty} \delta \tilde{Y}\left(\tau \wedge T_{k}\right)=0 P$-a.e., we have that $\lim _{k \rightarrow \infty} \delta \tilde{Y}\left(\tau \wedge T_{k}\right)=0$ in $L^{1}(\Omega)$. Letting $k \rightarrow \infty$ in (3.39) we get $|\delta \tilde{Y}(t)| \leq 0$. Hence $\tilde{Y}(t)=\tilde{Y}_{1}(t), t \geq 0$. From the equation satisfied by the solutions, we further deduce that $\int_{0}^{t}\left\langle\delta \tilde{Z}(s), d M_{s}\right\rangle=0$ and hence $E\left[\int_{0}^{t}\left\langle\delta \tilde{Z}(s), d M_{s}\right\rangle\right]^{2}=0$. This gives $\tilde{Z}=\tilde{Z}_{1}$. The proof is complete.

Let $f(x, y): D \times \mathbb{R} \rightarrow \mathbb{R}$ be a given Borel measurable function. Assume that $\mathrm{f}$ is continuous $w . r . t$. the variable $\mathrm{y}$ and satisfies

$$
\left\{\begin{array}{l}
\left(y_{1}-y_{2}\right)\left(f\left(x, y_{1}\right)-f\left(x, y_{2}\right)\right) \leq-J_{1}(x)\left|y_{1}-y_{2}\right|^{2}, \\
|f(x, y)| \leq C\left(1+\left|J_{1}(x)\right|+J_{2}(x)|y|\right),
\end{array}\right.
$$


where $J_{1}(x), J_{2}(x) \in L^{p}(D)$ and $\mathrm{C}$ is a constant. We have the following important corollary:

Corollary 3.3 Assume there exists a $x_{0} \in D$ such that

$$
E_{x_{0}}\left[e^{-\int_{0}^{\tau} D J_{1}(X(s)) d s}\right]<\infty .
$$

Then for any $\mathcal{F}_{\tau_{D}}$-measurable bounded r.v. $\xi$ and $x \in D$, the following two statements hold:

(i) There exists a unique pair $\left(Y_{x}, Z_{x}\right)$ satisfying the following BSDE:

$$
Y_{x}(t)=\xi+\int_{t \wedge \tau_{D}}^{\tau_{D}} f\left(X(s), Y_{x}(s)\right) d s-\int_{t \wedge \tau_{D}}^{\tau_{D}}\left\langle Z_{x}(s), d M_{s}\right\rangle, \quad P_{x} \text {-a.e., }
$$

such that $e^{-\int_{0}^{t} J_{1}(X(s)) d s} Y_{x}(t) \in$ Class $(D)$. Furthermore,

$$
\sup _{x \in D} Y_{x}(0)<\infty .
$$

(ii) There exists at most one solution $\left(Y_{x}, Z_{x}\right)$ satisfying the BSDE (3.41) such that $Y_{x}$ is a bounded process.

Remark 3.1 Please be ware of the subtle difference of (i) and (ii). We do not assume $e^{-\int_{0}^{t} J_{1}(X(s)) d s} Y_{x}(t) \in$ Class (D) in (ii). The statement (ii) is needed in the next section.

Proof (i) Since $J_{1}, J_{2} \in L^{p}(D)$, we know from (3.6) that $E_{x}\left[\int_{0}^{\tau_{D}}\left(\left|J_{1}(X(s))\right|+\right.\right.$ $\left.\left.J_{2}(X(s))\right) d s\right]<\infty$. By Lemma 3.1 and (3.40) we see that all the conditions in Theorem 3.2 are satisfied. Hence for any $x \in D$, there exists a unique solution $\left(Y_{x}, Z_{x}\right)$ to (3.41) such that $e^{-\int_{0}^{t} J_{1}(X(s)) d s} Y_{x}(t) \in$ Class (D).

Next we prove the last statement of the Corollary (i).

Set $\tilde{Y}_{x}(t)=e^{-\int_{0}^{t} J_{1}(X(s)) d s} Y_{x}(t), \tilde{Z}_{x}(t)=e^{-\int_{0}^{t} J_{1}(X(s)) d s} Z_{x}(t)$ and $\tilde{f}(t, y)=$ $J_{1}(X(t)) y+$

$e^{-\int_{0}^{t} J_{1}(X(s)) d s} f\left(X(t), e^{\int_{0}^{t} J_{1}(X(s)) d s} y\right), \tilde{\xi}=e^{-\int_{0}^{\tau} J_{1}(X(s)) d s} \xi$ as in Theorem 2.1. It follows from (3.41) that

$$
\tilde{Y}_{x}(t)=\tilde{\xi}+\int_{t \wedge \tau_{D}}^{\tau_{D}} \tilde{f}\left(s, \tilde{Y}_{x}(s)\right) d s-\int_{t \wedge \tau_{D}}^{\tau_{D}}\left\langle\tilde{Z}_{x}(s), d M_{s}\right\rangle .
$$

Choose a sequence of stopping times $\left\{\tau_{n}\right\}$ that increases to $\infty$ and such that $\int_{0}^{t \wedge \tau_{n}}\left\langle\frac{\tilde{Y}_{x}(s)}{\left|\tilde{Y}_{x}(s)\right|} \tilde{Z}_{x}(s), d M_{s}\right\rangle$ is a uniformly integrable martingale under $P_{x}$. By Lemma 3.2, it follows from (3.42) that

$$
\begin{aligned}
\left|\tilde{Y}_{x}\left(t \wedge \tau_{n}\right)\right| & =\left|\tilde{Y}_{x}\left(\tau_{D} \wedge \tau_{n}\right)\right|+\int_{t \wedge \tau_{D} \wedge \tau_{n}}^{\tau_{0} \wedge \tau_{n}} \frac{\tilde{Y}_{x}(s)}{\left|\tilde{Y}_{x}(s)\right|} \tilde{f}\left(s, \tilde{Y}_{x}(s)\right) d s-\int_{t \wedge \tau_{D} \wedge \tau_{n}}^{\tau_{D} \wedge \tau_{n}}\left\langle\frac{\tilde{Y}_{X}(s)}{\left|\tilde{Y}_{x}(s)\right|} \tilde{Z}_{x}(s), d M_{s}\right\rangle \\
& \leq\left|\tilde{Y}_{x}\left(\tau_{D} \wedge \tau_{n}\right)\right|-\int_{t \wedge \tau_{D} \wedge \tau_{n}}^{\tau_{\tau \wedge \tau_{n}}}\left\langle\frac{\tilde{Y}_{x}(s)}{\left|\tilde{Y}_{x}(s)\right|} \tilde{Z}_{x}(s), d M_{s}\right\rangle .
\end{aligned}
$$

Taking the conditional expectation with respect to $\mathcal{F}_{t}$ on both sides of the above inequality, we have

$$
\left|\tilde{Y}_{x}\left(t \wedge \tau_{n}\right)\right| \leq E_{x}\left[\left|\tilde{Y}_{x}\left(\tau \wedge \tau_{n}\right)\right| \mid \mathcal{F}_{t}\right] .
$$

Since $\tilde{Y}_{x}(t) \in$ Class (D), letting $n \rightarrow \infty$ in (3.43) we get

$$
\left|\tilde{Y}_{x}(t)\right| \leq E_{x}\left[|\tilde{\xi}| \mid \mathcal{F}_{t}\right]
$$


In particular, we have

$$
\begin{aligned}
& \sup _{x \in D}\left|Y_{x}(0)\right|=\sup _{x \in D}\left|\tilde{Y}_{x}(0)\right| \leq \sup _{x \in D} E_{x}[|\tilde{\xi}|]
\end{aligned}
$$

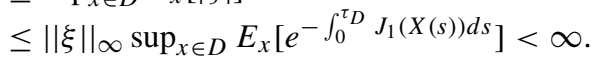

(ii) Suppose that $\left(Y_{x}, Z_{x}\right)$ and $\left(Y_{x}^{\prime}, Z_{x}^{\prime}\right)$ are solutions to BSDE (3.41) such that $Y_{x}$ and $Y_{x}^{\prime}$ are both bounded processes. Since $J_{1} \in L^{p}(D)$, there exists constant $\alpha>0, \gamma>1$ such that $\left\|\gamma\left(-J_{1}-\alpha\right)^{+}\right\|_{L^{p}(D)}<\frac{1}{\sigma}$ where $\sigma$ is defined in (3.6). Then by (3.6) and Kahamiskii's

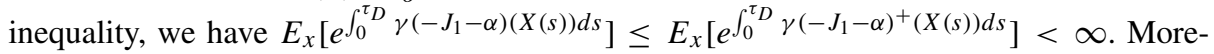
over by (3.7) and (3.8)

$$
\gamma_{1}<E_{x}\left[e^{\int_{0}^{\tau} D} \gamma\left(-J_{1}-\alpha\right)(X(s)) d s\right]<\gamma_{2}, \quad \forall x \in \bar{D},
$$

for some positive constants $\gamma_{1}, \gamma_{2}>0$.

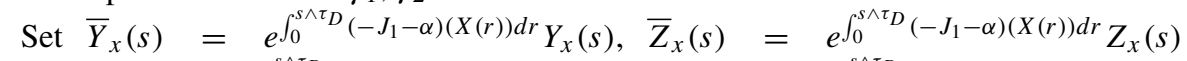

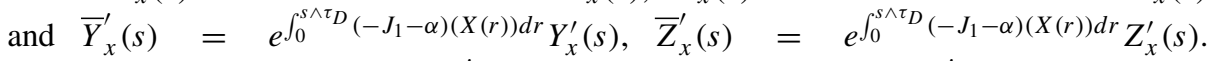
Set $\delta \bar{Y}_{x}(s)=\bar{Y}_{x}(s)-\bar{Y}_{x}^{\prime}(s), \delta \bar{Z}_{x}(s)=\bar{Z}_{x}(s)-\bar{Z}_{x}^{\prime}(s), \bar{f}(s, y)=$ $e^{\int_{0}^{s}\left(-J_{1}-\alpha\right)(X(r)) d r} f\left(X(s), e^{\int_{0}^{s}\left(J_{1}+\alpha\right)(X(r)) d r} y\right)+\left(J_{1}+\alpha\right)(X(s)) y$. Then

$$
\left(y_{1}-y_{2}\right)\left(\bar{f}\left(s, y_{1}\right)-\bar{f}\left(s, y_{2}\right)\right) \leq \alpha\left(y_{1}-y_{2}\right)^{2} .
$$

Choose a sequence of stopping times $\left\{\tau_{n}^{\prime}\right\}_{n \geq 1}$ that increases to $\infty$ and such that $\int_{0}^{t \wedge \tau_{n}^{\prime} \wedge \tau_{D}}\left\langle\frac{\delta \bar{Y}_{x}(s)}{\left|\delta \bar{Y}_{x}(s)\right|} \delta \bar{Z}_{x}(s), d M_{s}\right\rangle$ is a uniformly integrable martingale. By (3.45)

$$
\begin{aligned}
& \sup _{n} E_{X}\left[\left(e^{\int_{0}^{\tau_{n}^{\prime} \wedge \tau} D}\left(-J_{1}-\alpha\right)(X(s)) d s\right)^{\gamma}\right] \\
& \leq \sup _{n} \gamma_{1}^{-1} E_{x}\left[E_{X_{\tau_{n}^{\prime} \wedge \tau_{D}}}\left[e^{\int_{0}^{\tau} D} \gamma\left(-J_{1}-\alpha\right)(X(s)) d s\right] \cdot e^{\int_{0}^{\tau_{n}^{\prime} \wedge \tau_{D}} \gamma\left(-J_{1}-\alpha\right)(X(s)) d s}\right] \\
& =\sup _{n} \gamma_{1}^{-1} E_{x}\left[e^{\int_{0}^{\tau_{n} \wedge} \gamma\left(-J_{1}-\alpha\right)(X(s)) d s}\right] \leq \gamma_{1}^{-1} \gamma_{2}<\infty \text {, }
\end{aligned}
$$

which implies that $\left\{e^{\int_{0}^{\tau_{n}^{\prime} \wedge \tau} D}\left(-J_{1}-\alpha\right)(X(s)) d s\right\}_{n \geq 1}$ is $P_{x}$-uniformly integrable. Hence $\left\{\delta \bar{Y}_{x}\left(\tau_{n}^{\prime} \wedge\right.\right.$ $\left.\left.\tau_{D}\right)\right\}_{n \geq 1}$ is $P_{x}$-uniformly integrable because $Y_{x}-Y_{x}^{\prime}$ is bounded. Similarly, we can show that $\left\{\delta \bar{Y}_{x}\left(t \wedge \tau_{n}^{\prime} \wedge \tau_{D}\right)\right\}_{n \geq 1}$ is also $P_{x}$-uniformly integrable.

Note that

$$
\delta \bar{Y}_{x}(t)=\int_{t \wedge \tau_{D}}^{\tau_{D}}\left(\bar{f}\left(s, \bar{Y}_{x}(s)\right)-\bar{f}\left(s, \bar{Y}_{x}^{\prime}(s)\right)\right) d s-\int_{t \wedge \tau_{D}}^{\tau_{D}}\left\langle\delta \bar{Z}(s), d M_{s}\right\rangle .
$$

By Lemma 3.2 and (3.46), it holds that

$$
\begin{aligned}
& \left|\delta \bar{Y}_{x}\left(t \wedge \tau_{n}^{\prime} \wedge \tau_{D}\right)\right| \\
\leq & \left|\delta \bar{Y}_{x}\left(\tau_{n}^{\prime} \wedge \tau_{D}\right)\right|+\int_{t \wedge \tau_{n}^{\prime} \wedge \tau_{D}}^{\tau_{n}^{\prime}} \frac{\delta \bar{Y}_{x}(s)}{\left|\delta \bar{Y}_{x}(s)\right|}\left(\bar{f}\left(s, \bar{Y}_{x}(s)\right)-\bar{f}\left(s, \bar{Y}_{x}^{\prime}(s)\right)\right) d s \\
& -\int_{t \wedge \tau_{n}^{\prime} \wedge \tau_{D}}^{\tau_{n}^{\prime} \wedge \tau_{D}}\left\langle\frac{\delta \bar{Y}_{x}(s)}{\left|\delta \bar{Y}_{x}(s)\right|} \delta Z(s), d M_{s}\right\rangle \\
\leq & \left|\delta \bar{Y}_{x}\left(\tau_{n}^{\prime} \wedge \tau_{D}\right)\right|+\alpha \int_{t \wedge \tau_{n}^{\prime} \wedge \tau_{D}}^{\tau_{n}^{\prime} \wedge \tau_{D}}\left|\delta \bar{Y}_{x}(s)\right| d s \\
& -\int_{t \wedge \tau_{n}^{\prime} \wedge \tau_{D}}^{\tau_{n}^{\prime} \wedge \tau_{D}}\left\langle\frac{\delta \bar{Y}_{x}(s)}{\left|\delta \bar{Y}_{x}(s)\right|} \delta Z(s), d M_{s}\right\rangle, \quad P_{x}-\text { a.e.. }
\end{aligned}
$$

Taking the expectation on the both sides of (3.47) we get

$$
E_{x}\left[\left|\delta \bar{Y}_{x}\left(t \wedge \tau_{n}^{\prime} \wedge \tau_{D}\right)\right|\right] \leq E_{x}\left[\left|\delta \bar{Y}_{x}\left(\tau_{n}^{\prime} \wedge \tau_{D}\right)\right|\right]+\alpha E_{x}\left[\int_{t \wedge \tau_{n}^{\prime} \wedge \tau_{D}}^{\tau_{n}^{\prime} \wedge \tau_{D}}\left|\delta \bar{Y}_{x}(s)\right| d s\right]
$$


Notice that $\lim _{n \rightarrow \infty}\left|\delta \bar{Y}_{x}\left(\tau_{n}^{\prime} \wedge \tau_{D}\right)\right|=e^{\int_{0}^{\tau} D}\left(-J_{1}-\alpha\right)(X(s)) d s|\xi-\xi|=0, P_{x}$-a.e.. Using the uniformly integrability of $\left\{\delta \bar{Y}_{x}\left(t \wedge \tau_{n}^{\prime} \wedge \tau_{D}\right)\right\}_{n \geq 1}$ and $\left\{\delta \bar{Y}_{x}\left(\tau_{n}^{\prime} \wedge \tau_{D}\right)\right\}_{n \geq 1}$, letting $n \rightarrow \infty$ in (3.48) we find

$$
E_{x}\left[\left|\delta \bar{Y}_{x}(t)\right|\right] \leq \alpha \int_{t}^{\infty} E_{x}\left[\left|\delta \bar{Y}_{x}(s)\right|\right] d s .
$$

By Gronwall's inequality we conclude $E_{x}\left[\left|\delta \bar{Y}_{x}(t)\right|\right]=0$. Hence $Y_{x}=Y_{x}^{\prime}$. Furthermore we deduce that $\int_{0}^{t}\left\langle\delta \bar{Z}_{x}(s), d M_{s}\right\rangle=0$ and hence $Z_{x}=Z_{x}^{\prime}$. The uniqueness is proved.

\section{Dirichlet Boundary Value Problem}

\subsection{Linear Case}

Consider the following second order elliptic operator

$$
L_{2} u=\frac{1}{2} \sum_{i, j=1}^{d} \frac{\partial}{\partial x_{i}}\left(a_{i j}(x) \frac{\partial u}{\partial x_{j}}\right)+\sum_{i=1}^{d} b_{i}(x) \frac{\partial u}{\partial x_{i}}+q(x) .
$$

The quadratic form generated by $L_{2}$ is

$$
\left\{\begin{aligned}
\mathcal{E}^{q}(u, v) & =\left(-L_{2} u, v\right) \\
& =\frac{1}{2} \sum_{i, j=1}^{d} \int_{D} a_{i j}(x) \frac{\partial u}{\partial x_{i}} \frac{\partial v}{\partial x_{j}} d x-\sum_{i=1}^{d} \int_{D} b_{i}(x) \frac{\partial u}{\partial x_{i}} v(x) d x-\int_{D} q(x) u(x) v(x) d x, \\
D\left(\mathcal{E}^{q}\right) & =W_{0}^{1,2}(D) .
\end{aligned}\right.
$$

Since $|b|^{2}, q \in L^{p}$ for $p>\frac{d}{2}$, it is known that $\left(\mathcal{E}^{q}, D\left(\mathcal{E}^{q}\right)\right)$ is a coercive closed form and there exist a $\alpha_{0}>0$ such that

$$
\lambda_{\alpha_{0}} \mathcal{E}(u, u) \leq \mathcal{E}_{\alpha_{0}}^{q}(u, u) \leq \Lambda_{\alpha_{0}} \mathcal{E}(u, u)
$$

for some constant $\lambda_{\alpha_{0}}, \Lambda_{\alpha_{0}}$, where $\mathcal{E}(u, u)=\frac{1}{2} \sum_{i, j=1}^{d} \int_{D} \tilde{a}_{i j}(x) \frac{\partial u}{\partial x_{i}} \frac{\partial u}{\partial x_{j}} d x$ and $\mathcal{E}_{\alpha_{0}}^{q}(u, u)=$ $\mathcal{E}^{q}(u, u)+\alpha_{0}(u, u)$. By Theorem 3.2 in [13] or Lemma 4.1 in [7], for sufficient large $\alpha>0$, the $\alpha$-resolvent operator of $\left(\mathcal{E}^{q}, D\left(\mathcal{E}^{q}\right)\right)$ is given by

$$
G_{\alpha}^{q} f(x)=E_{x}\left[\int_{0}^{\tau_{D}} e^{\int_{0}^{t} q(X(s)) d s-\alpha t} f(X(t)) d t\right], \quad \forall f \in L^{2}(D),
$$

where $X=\left(X_{t}, P_{x}, x \in D\right)$ is the diffusion associated with the operator $L_{1}$ in (1.4).

Let $F$ be a measurable function satisfying

$$
|F(x)| \leq C+C|q(x)|,
$$

for some constant $C$. Take $\varphi \in C(\partial D)$ and consider the boundary value problem

$$
\begin{cases}L_{2} u(x)=-F(x), & \forall x \in D, \\ \left.u(x)\right|_{\partial D}=\varphi, & \forall x \in \partial D .\end{cases}
$$

We have the following result:

Theorem 4.1 Assume there exists a $x_{0} \in D$ such that

$$
E_{x_{0}}\left[\exp \left(\int_{0}^{\tau_{D}} q(X(s)) d s\right)\right]<\infty .
$$


Then there exists a unique continuous weak solution to problem (4.4) which is given by

$$
u(x)=E_{x}\left[e^{\int_{0}^{\tau_{D}} q(X(s)) d s} \varphi\left(X\left(\tau_{D}\right)\right)+\int_{0}^{\tau_{D}} e^{\int_{0}^{t} q(X(s)) d s} F(X(t)) d t\right] .
$$

Proof It is known from Lemma 2.1 in [5] that $u_{1}(x)=E_{x}\left[\varphi\left(X\left(\tau_{D}\right)\right)\right]$ is the unique continuous weak solution of the problem

$$
\begin{cases}L_{1} u_{1}(x)=0, & \forall x \in D, \\ \left.u_{1}(x)\right|_{\partial D}=\varphi, & \forall x \in \partial D .\end{cases}
$$

Let $F_{1}(x)=F(x)+q(x) u_{1}(x)$ and $u_{2}(x)=E_{x}\left[\int_{0}^{\tau_{D}} e^{\int_{0}^{t} q(X(s)) d s} F_{1}(X(t)) d t\right]$. By Lemma 3.1 and (4.3) $u_{2}$ is well defined. Let $u(x)$ be defined as (4.5). Then $u=u_{1}+u_{2}$. Hence, to prove that $u$ is a continuous weak solution to (4.4) we only need show that $u_{2}$ is a continuous weak solution of the following problem:

$$
\begin{cases}L_{2} u_{2}(x)=-F_{1}, & \forall x \in D \\ \left.u_{2}(x)\right|_{\partial D}=0, & \forall x \in \partial D .\end{cases}
$$

Since $u_{1}$ is bounded, by (4.3) we have $\left|F_{1}(x)\right| \leq c_{1}(1+|q(x)|)$ for some $c_{1}>0$. By Lemma 5.7 in [8] and Lemma 2.1 in [5], we know that the semigroup generated by $L_{2}$ is strong Feller. Hence, using the similar proof as that of Theorem 3.18 in [9] we obtain that $u_{2} \in C(\bar{D})$.

Next we show that $u_{2} \in W_{0}^{1,2}(D)$.

Since $q \in L^{p}$ for $p>\frac{d}{2}$ and since the transition density function of $\left(X_{t}, P_{x}, x \in \mathbb{R}^{d}\right)$ has the Gaussian upper bound estimate by (3.9), we can easily deduce that $\alpha G_{\alpha}^{q}$ is strongly continuous on $L^{p}$.

By the Markov property, it is easy to see that

$$
\alpha\left(u_{2}-\alpha G_{\alpha}^{q} u_{2}\right)=\alpha G_{\alpha}^{q} F_{1}(x) .
$$

Hence we get $\varlimsup_{\alpha \rightarrow \infty} \alpha\left(u_{2}-\alpha G_{\alpha}^{q} u_{2}, u_{2}\right) \leq \varlimsup_{\alpha \rightarrow \infty}\left\|\alpha G_{\alpha}^{q} F_{1}(x)\right\|_{L^{p}}\left\|u_{2}\right\|_{L^{p^{\prime \prime}}}<\infty$, where $\frac{1}{p}+\frac{1}{p^{\prime \prime}}=1$. By Theorem 1.1.4 in [15] we deduce that $u_{2} \in W_{0}^{1,2}(D)$.

Finally we show that $u_{2}$ is a continuous weak solution of (4.7). For any $\phi \in C_{0}^{\infty}(D)$, by (4.8) we have

$$
\mathcal{E}^{q}\left(u_{2}, \phi\right)-\left(F_{1}, \phi\right)=\lim _{\alpha \rightarrow \infty}\left(\alpha\left(u_{2}-\alpha G_{\alpha}^{q} u_{2}, F_{1}\right)-\left(F_{1}, \phi\right)=\lim _{\alpha \rightarrow \infty}\left(\alpha G_{\alpha}^{q} F_{1}, \phi\right)-\left(F_{1}, \phi\right)=0 .\right.
$$

Hence $u_{2}$ is a continuous weak solution of (4.7).

Now we prove the uniqueness. If $\tilde{u}$ is another continuous weak solution of (4.4), then $\tilde{u}-u_{2}$ is a continuous weak solution of (4.6). By the uniqueness of (4.6), we get $\tilde{u}=u$.

\subsection{Semilinear Case}

Recall that $M^{0}$ is the martingale part of the diffusion process $\left(X_{t}, P_{x}^{0}\right)$ generated by $L_{0}$. Define the exponential martingale

$$
U(t)=\exp \left(\int_{0}^{t}\left\langle\tilde{a}^{-1} b(X(s)), d M_{s}^{0}\right\rangle-\frac{1}{2} \int_{0}^{t}\left\langle\tilde{a}^{-1} b(X(s)), b(X(s))\right\rangle d s\right) .
$$

Then we have

$$
\left.\frac{d P_{x}}{d P_{x}^{0}}\right|_{\mathcal{F}_{t}}=U(t)
$$


Let $g(x, y): D \times \mathbb{R} \rightarrow \mathbb{R}$ be a measurable function such that $g$ is continuous w.r.t. $y$ and satisfies

$$
\left\{\begin{array}{l}
\left(y_{1}-y_{2}\right)\left(g\left(x, y_{1}\right)-g\left(x, y_{2}\right)\right) \leq-K(x)\left|y_{1}-y_{2}\right|^{2}, \\
|g(x, y)| \leq C\left(1+|q(x)-K(x)|+\left|K_{1}(x) y\right|\right),
\end{array}\right.
$$

for some constant $C$ and $K, K_{1} \in L^{p}(D)$.

Consider the following semilinear boundary value problem:

$$
\begin{cases}L_{2} u(x)=-g(x, u(x)), & \forall x \in D \\ \left.u(x)\right|_{\partial D}=\varphi, & \forall x \in \partial D\end{cases}
$$

Lemma 4.1 Assume there exists a $x_{0} \in D$ such that

$$
\begin{aligned}
E_{x_{0}}^{0}[ & \exp \left(\int_{0}^{\tau_{D}}\left\langle\widetilde{a}^{-1} b(X(s)), d M_{s}^{0}\right\rangle-\frac{1}{2} \int_{0}^{\tau_{D}}\left(b^{*} \widetilde{a}^{-1} b\right)(X(s)) d s\right. \\
& \left.\left.+\int_{0}^{\tau_{D}}(q(X(s))-K(X(s))) d s\right)\right]<\infty .
\end{aligned}
$$

Then there exists a unique continuous weak solution to the problem (4.9).

Proof Set $g_{1}(x, y)=g(x, y)+q(x) y$. By (4.10) and Corollary 3.3 for any $x \in D$, there exists a unique solution $\left(Y_{x}, Z_{x}\right)$ to the following BSDE:

$$
Y_{x}(t)=\varphi\left(X\left(\tau_{D}\right)\right)+\int_{t \wedge \tau_{D}}^{\tau_{D}} g_{1}\left(X(s), Y_{x}(s)\right) d s-\int_{t \wedge \tau_{D}}^{\tau_{D}}\left\langle Z_{x}(s), d M_{s}\right\rangle, \quad P_{x} \text {-a.e.. }
$$

Define $u_{0}(x):=Y_{x}(0), v_{0}(x):=Z_{x}(0)$. By the strong Markov property of $\mathrm{X}$ and the uniqueness of the $\operatorname{BSDE}(4.11)$, it is easy to see that $P_{x}$-a.e.

$$
u_{0}(X(t))=Y_{x}(t), \quad v_{0}(X(t))=Z_{x}(t), \quad 0 \leq t \leq \tau_{D} .
$$

Consider the following linear problem:

$$
\begin{cases}L_{1} u(x)=-g_{1}\left(x, u_{0}(x)\right), & \forall x \in D \\ \left.u(x)\right|_{\partial D}=\varphi, & \forall x \in \partial D .\end{cases}
$$

Since $u_{0}$ is bounded by Corollary 3.3 , by Theorem 4.1 the above equation admits a unique continuous weak solution $u \in W^{1,2}(D)$. On the other hand, by the Fukushima's decomposition we have for q.e. $x \in D, P_{x}$-a.e.

$$
\begin{aligned}
u(X(t))-u(x) & =\int_{0}^{t}\left\langle\nabla u(X(s)), d M_{s}\right\rangle+\int_{0}^{t} g_{1}\left(X(s), u_{0}(X(s))\right) d s \\
& =\int_{0}^{t}\left\langle\nabla u(X(s)), d M_{s}\right\rangle+\int_{0}^{t} g_{1}\left(X(s), Y_{x}(s)\right) d s, \quad \forall t<\tau_{D} .
\end{aligned}
$$

Choose a sequence of stopping times $\left\{T_{n}\right\}_{n \geq 1}$ that increases to $\infty$ and such that $\int_{0}^{t \wedge T_{n} \wedge \tau_{D}}\left\langle Z_{x}(s)\right.$

$\left.d M_{S}\right\rangle$ and $\int_{0}^{t \wedge T_{n} \wedge \tau_{D}}\left\langle\nabla u(X(s)), d M_{S}\right\rangle$ are $P_{x}$-uniformly integrable martingales. It follows from (4.11) and (4.13) that

$$
\begin{gathered}
Y_{x}\left(t \wedge \tau_{n} \wedge \tau_{D}\right)-Y_{X}\left(\tau_{n} \wedge \tau_{D}\right)=\int_{t \wedge \tau_{n} \wedge \tau_{D}}^{\tau_{n} \wedge \tau_{D}} g_{1}\left(X(s), Y_{x}(s)\right) d s-\int_{t \wedge \tau_{n} \wedge \tau_{D}}^{\tau_{n} \wedge \tau_{D}}\left\langle Z_{x}(s), d M_{s}\right\rangle, \\
u\left(X\left(t \wedge T_{n} \wedge \tau_{D}\right)\right)-u\left(X\left(T_{n} \wedge \tau_{D}\right)\right)=\int_{t \wedge T_{n} \wedge \tau_{D}}^{T_{n} \wedge \tau_{D}} g_{1}\left(X(s), Y_{x}(s)\right) d s-\int_{t \wedge T_{n} \wedge \tau_{D}}^{T_{n} \wedge \tau_{D}}\left\langle\nabla u(X(s)), d M_{s}\right\rangle .
\end{gathered}
$$


Taking the conditional expectations respect to $\mathcal{F}_{t}$ on both sides of the above equations, we get

$$
u\left(X\left(t \wedge T_{n} \wedge \tau_{D}\right)\right)-E_{x}\left[u\left(X\left(T_{n} \wedge \tau_{D}\right)\right) \mid \mathcal{F}_{t}\right]=Y_{x}\left(t \wedge T_{n} \wedge \tau_{D}\right)-E_{x}\left[Y_{x}\left(T_{n} \wedge \tau_{D}\right) \mid \mathcal{F}_{t}\right] .
$$

Since $u \in C(\bar{D})$ and since $Y_{x}(t)$ is bounded (see (4.12)), let $T_{n} \rightarrow \infty$ in (4.14) to obtain

$$
u\left(X\left(t \wedge \tau_{D}\right)\right)-E_{x}\left[u\left(\left(X\left(\tau_{D}\right)\right) \mid \mathcal{F}_{t}\right]=Y_{x}\left(t \wedge \tau_{D}\right)-E_{x}\left[\varphi\left(\tau_{D}\right) \mid \mathcal{F}_{t}\right] .\right.
$$

Since $\left.u(x)\right|_{\partial D}=\varphi$, letting $t=0$ we deduce that $u(x)=Y_{x}(0)=u_{0}(x)$ for q.e. $x \in D$. Hence $u$ is a weak solution to (4.9).

Now we proceed to prove the uniqueness of the boundary value problem (4.9). Suppose $u_{1}$ is another continuous weak solution of (4.9). Then by Fukushima's decomposition $\left(u_{1}(X(t)), \nabla u_{1}(X(t))\right)$ is also a solution of BSDE (4.11) for q.e. $x \in D$. Since $u(X(t))$ and $u_{1}(X(t))$ are bounded, by Corollary 3.3(ii) we have $u_{1}(X(t))=u(X(t))$. In particular, $\left|u_{1}(x)-u(x)\right|=E_{x}\left[\left|u_{1}(X(0))-u(X(0))\right|\right]=0$ for q.e. $x \in D$. By the continuity of $u_{1}$ and $u$, it holds for every $x \in D$. The uniqueness is proved.

Let $f(x, y): D \times \mathbb{R} \rightarrow \mathbb{R}$ be a measurable function that is continuous w.r.t. $y$ and satisfies

$$
\left\{\begin{array}{l}
\left(y_{1}-y_{2}\right)\left(f\left(x, y_{1}\right)-f\left(x, y_{2}\right)\right) \leq-J(x)\left|y_{1}-y_{2}\right|^{2}, \\
|f(x, y)| \leq C\left(1+J_{1}(x)|y|\right),
\end{array}\right.
$$

for some constant $C$ and $J, J_{1} \in L^{p}(D)$. Consider the following semilinear elliptic PDEs:

$$
\begin{cases}\mathcal{A} u(x)=-f(x, u(x)), & \forall x \in D, \\ \left.u(x)\right|_{\partial D}=\varphi, & \forall x \in \partial D .\end{cases}
$$

Now we can state the main result in this section.

Theorem 4.2 Under the Assumption I, if there exists a $x_{0} \in D$ such that

$$
\begin{aligned}
E_{x_{0}}^{0}[ & \exp \left(\int_{0}^{\tau_{D}}\left\langle\widetilde{a}^{-1}(b-\hat{b})(X(s)), d M_{s}^{0}\right\rangle-\frac{1}{2} \int_{0}^{\tau_{D}}(b-\hat{b})^{*} \widetilde{a}^{-1}(b-\hat{b})(X(s)) d s\right. \\
& \left.\left.+N_{\tau_{D}}^{v}+\int_{0}^{\tau_{D}}(q(X(s))-J(X(s))) d s\right)\right]<\infty,
\end{aligned}
$$

where $E_{x_{0}}^{0}$ stands for the expectation under $P_{x_{0}}^{0}, N_{t}^{v}$ is the zero-energy part of the Fukushima's decomposition defined in (2.2). Then there exists a unique continuous weak solution to problem (4.16). Moreover, if $f(x, y)=F(x)$, the solution is given by

$$
\begin{aligned}
u(x)= & E_{x}^{0}\left[\operatorname { e x p } \left(\int_{0}^{\tau_{D}}\left\langle\widetilde{a}^{-1}(b-\hat{b})(X(s)), d M_{s}^{0}\right\rangle-\frac{1}{2} \int_{0}^{\tau_{D}}\left((b-\hat{b})^{*} \tilde{a}^{-1}(b-\hat{b})\right)(X(s)) d s\right.\right. \\
& \left.\left.+N_{\tau_{D}}^{v}+\int_{0}^{\tau_{D}} q(X(s)) d s\right) \varphi\left(X\left(\tau_{D}\right)\right)\right] \\
& +E_{x}^{0}\left[\int _ { 0 } ^ { \tau _ { D } } \operatorname { e x p } \left(\int_{0}^{t}\left\langle\widetilde{a}^{-1}(b-\hat{b})(X(s)), d M_{s}^{0}\right\rangle-\frac{1}{2} \int_{0}^{t}\left((b-\hat{b})^{*} \widetilde{a}^{-1}(b-\hat{b})\right)(X(s)) d s\right.\right. \\
& \left.\left.+N_{t}^{v}+\int_{0}^{t} q(X(s)) d s\right) F(X(t)) d t\right] .
\end{aligned}
$$

Proof Introduce the following operator on $L^{2}(D)$ :

$$
\begin{aligned}
\hat{\mathcal{A}}= & \frac{1}{2} \sum_{i, j=1}^{d} \frac{\partial}{\partial x_{i}}\left(a_{i j}(x) \frac{\partial}{\partial x_{j}}\right)+\sum_{i=1}^{d}\left[b_{i}-\hat{b}_{i}-(\tilde{a} \nabla v)_{i}\right] \frac{\partial}{\partial x_{i}} \\
& +\frac{1}{2}(\nabla v)^{*} \tilde{a} \nabla v-(b-\hat{b})^{*} \nabla v+q .
\end{aligned}
$$

Set $h=e^{v}$ and consider the following semi-linear elliptic PDE:

$$
\begin{cases}\hat{\mathcal{A}} \hat{u}(x)=-h(x) f\left(x, h^{-1}(x) \hat{u}(x)\right), & \forall x \in D, \\ \left.\hat{u}(x)\right|_{\partial D}=h(x) \varphi(x), & \forall x \in \partial D .\end{cases}
$$


Note that:

$$
\begin{aligned}
& \int_{0}^{\tau_{D}}\left\langle\widetilde{a}^{-1}(b-\hat{b}-\tilde{a} \nabla v)(X(s)), d M_{s}^{0}\right\rangle-\frac{1}{2} \int_{0}^{\tau_{D}}\left((b-\hat{b}-\tilde{a} \nabla v)^{*} \tilde{a}^{-1}(b-\hat{b}-\tilde{a} \nabla v)\right)(X(s)) d s \\
& +\int_{0}^{\tau_{D}}\left(\frac{1}{2}(\nabla v)^{*} \tilde{a} \nabla v-(b-\hat{b})^{*} \nabla v+q-J\right)(X(s)) d s \\
= & \int_{0}^{\tau_{D}}\left\langle\widetilde{a}^{-1}(b-\hat{b})(X(s)), d M_{s}^{0}\right\rangle-\int_{0}^{\tau_{D}}\left\langle\tilde{a} \nabla v, d M_{s}^{0}\right\rangle-\frac{1}{2} \int_{0}^{\tau_{D}}\left((b-\hat{b})^{*} \widetilde{a}^{-1}(b-\hat{b})(X(s)) d s\right. \\
& +\frac{1}{2} \int_{0}^{\tau_{D}}(b-\hat{b})^{*}(\nabla v)(X(s)) d s+\frac{1}{2} \int_{0}^{\tau_{D}}(\nabla v)^{*}(b-\hat{b}-\tilde{a} \nabla v)(X(s)) d s \\
& +\int_{0}^{\tau_{D}}\left(\frac{1}{2}(\nabla v)^{*} \tilde{a} \nabla v-(b-\hat{b})^{*} \nabla v+q-J\right)(X(s)) d s \\
= & \int_{0}^{\tau_{D}}\left\langle\tilde{a}^{-1}(b-\hat{b})(X(s)), d M_{s}^{0}\right\rangle-\frac{1}{2} \int_{0}^{\tau_{D}}\left((b-\hat{b})^{*} \widetilde{a}^{-1}(b-\hat{b})(X(s)) d s-M_{\tau_{D}}^{v}\right. \\
& +\int_{0}^{\tau_{D}}(q-J)(X(s)) d s \\
= & \int_{0}^{\tau_{D}}\left\langle\widetilde{a}^{-1}(b-\hat{b})(X(s)), d M_{s}^{0}\right\rangle-\frac{1}{2} \int_{0}^{\tau_{D}}\left((b-\hat{b})^{*} \widetilde{a}^{-1}(b-\hat{b})(X(s)) d s\right. \\
& +N_{\tau_{D}}^{v}+v(X(0))-v\left(X\left(\tau_{D}\right)\right)+\int_{0}^{\tau_{D}}(q-J)(X(s)) d s .
\end{aligned}
$$

Thus condition (4.17) is equivalent to

$$
\begin{aligned}
E_{x_{0}}[ & \exp \left(\int_{0}^{\tau_{D}}\left\langle\widetilde{a}^{-1}(b-\hat{b}-\tilde{a} \nabla v)(X(s)), d M_{s}^{0}\right\rangle\right. \\
& \quad-\frac{1}{2} \int_{0}^{\tau_{D}}\left((b-\hat{b}-\tilde{a} \nabla v)^{*} \widetilde{a}^{-1}(b-\hat{b}-\tilde{a} \nabla v)\right)(X(s)) d s \\
& \left.\left.+\int_{0}^{\tau_{D}}\left(\frac{1}{2}(\nabla v)^{*} \tilde{a} \nabla v-(b-\hat{b})^{*} \nabla v+q-J\right)(X(s)) d s\right)\right]<\infty .
\end{aligned}
$$

Since $h(x)>0,(4.15)$ implies

$$
\left\{\begin{array}{l}
\left(y_{1}-y_{2}\right)\left(h(x) f\left(x, h^{-1}(x) y_{1}\right)-h(x) f\left(x, h^{-1}(x) y_{2}\right)\right) \leq-J(x)\left|y_{1}-y_{2}\right|^{2}, \\
\mid\left(h(x) f\left(x, h^{-1}(x) y\right) \mid \leq C\left(\sup _{x \in D} h(x)+\left|J_{1}(x) y\right|\right) .\right.
\end{array}\right.
$$

By (4.21), (4.22) and Lemma 4.1, we conclude that there exists a unique continuous weak solution $\hat{u}$ to problem (4.19). Let $u(x)=h^{-1}(x) \hat{u}(x)$. Now following the same arguments as in the proof of Theorem 5.1 in [18], we can show that $u$ is the continuous weak solution of problem (4.16). We omit the details here.

Let us now consider the special case, $f(x, y)=F(x)$. Define $\hat{U}(t)=\exp \left(\int_{0}^{t}\left\langle\widetilde{a}^{-1}(b-\right.\right.$ $\left.\left.\hat{b}-\tilde{a} \nabla v)(X(s)), d M_{s}^{0}\right\rangle-\frac{1}{2} \int_{0}^{t}\left((b-\hat{b}-\tilde{a} \nabla v)^{*} \tilde{a}^{-1}(b-\hat{b}-\tilde{a} \nabla v)\right)(X(s)) d s\right)$. Since $b-$ $\hat{b}-\tilde{a} \nabla v \in L^{2 p}(D)$, by Novikov's criterion $\hat{U}(t)$ is a $P_{x}^{0}$-exponential martingale. Define

$$
\left.\frac{d \hat{P}_{x}}{d P_{x}^{0}}\right|_{\mathcal{F}_{t}}=\hat{U}(t)
$$

Then it follows from Theorem 4.1 that the solution to problem (4.19) admits the representation:

$$
\begin{aligned}
\hat{u}(x)= & \hat{E}_{x}\left[e^{\int_{0}^{\tau} \tau_{D}^{x}\left(\frac{1}{2}(\nabla v)^{*} \tilde{a} \nabla v-(b-\hat{b})^{*} \nabla v+q\right)(X(s)) d s} \varphi\left(X\left(\tau_{D}\right)\right)\right] \\
& +\hat{E}_{x}\left[\int_{0}^{\tau_{D}} e^{\int_{0}^{t}\left(\frac{1}{2}(\nabla v)^{*} \tilde{a} \nabla v-(b-\hat{b})^{*} \nabla v+q\right)(X(s)) d s} h(X(t)) F(X(t)) d t\right] \\
& :=\hat{u}_{1}(x)+\hat{u}_{2}(x),
\end{aligned}
$$

where $\hat{E}_{x}$ is the expectation under $\hat{P}_{x}$. By the calculations in (4.20), we have

$$
\begin{aligned}
\hat{u}_{1}(x)= & E_{x}^{0}\left[\hat{U}_{\tau_{D^{x}}} e^{\int_{0}^{\tau_{D}^{x}}\left(\frac{1}{2}(\nabla v)^{*} \tilde{a} \nabla v-(b-\hat{b})^{*} \nabla v+q\right)(X(s)) d s} \varphi\left(X\left(\tau_{D}\right)\right)\right] \\
= & E_{x}^{0}\left[\operatorname { e x p } \left(\int_{0}^{\tau_{D}}\left\langle\widetilde{a}^{-1}(b-\hat{b})(X(s)), d M_{s}^{0}\right\rangle-\frac{1}{2} \int_{0}^{\tau_{D}}\left((b-\hat{b})^{*} \widetilde{a}^{-1}(b-\hat{b})\right)(X(s)) d s\right.\right. \\
& \left.\left.\quad+N_{\tau_{D}}^{v}+\int_{0}^{\tau_{D}} q(X(s)) d s\right) \varphi\left(X\left(\tau_{D}\right)\right)\right] e^{v(x)},
\end{aligned}
$$


and

$$
\begin{aligned}
\hat{u}_{2}(x)= & E_{x}^{0}\left[\int_{0}^{\tau_{D}} \hat{U}(t) e^{\int_{0}^{t}\left(\frac{1}{2}(\nabla v)^{*} \tilde{a} \nabla v-(b-\hat{b})^{*} \nabla v+q\right)(X(s)) d s} h(X(t)) F(X(t)) d t\right] \\
= & E_{x}^{0}\left[\int _ { 0 } ^ { \tau _ { D } } \operatorname { e x p } \left(\int_{0}^{\tau_{t}}\left\langle\widetilde{a}^{-1}(b-\hat{b})(X(s)), d M_{s}^{0}\right\rangle-\frac{1}{2} \int_{0}^{t}\left((b-\hat{b})^{*} \widetilde{a}^{-1}(b-\hat{b})\right)(X(s)) d s\right.\right. \\
& \left.\left.\quad+N_{t}^{v}+v(X(0))-v(X(t))+\int_{0}^{t} q(X(s)) d s h(X(t))\right) F(X(t)) d t\right] \\
= & E_{x}^{0}\left[\int _ { 0 } ^ { \tau _ { D } } \operatorname { e x p } \left(\int_{0}^{t}\left\langle\widetilde{a}^{-1}(b-\hat{b})(X(s)), d M_{s}^{0}\right\rangle-\frac{1}{2} \int_{0}^{t}\left((b-\hat{b})^{*} \widetilde{a}^{-1}(b-\hat{b})\right)(X(s)) d s\right.\right. \\
& \left.\left.\quad+N_{t}^{v}+\int_{0}^{t} q(X(s)) d s\right) F(X(t)) d t\right] e^{v(x)} .
\end{aligned}
$$

Hence the solution of (4.16) in this case is given by

$$
\begin{aligned}
u(x) & =h^{-1}(x) \hat{u}_{1}(x)+h^{-1}(x) \hat{u}_{2}(x) \\
& =E_{x}^{0}\left[\operatorname { e x p } \left(\int_{0}^{\tau_{D}}\left\langle\widetilde{a}^{-1}(b-\hat{b})(X(s)), d M_{s}^{0}\right\rangle-\frac{1}{2} \int_{0}^{\tau_{D}}\left((b-\hat{b})^{*} \widetilde{a}^{-1}(b-\hat{b})\right)(X(s)) d s\right.\right. \\
& \left.\left.+N_{\tau_{D}}^{v}+\int_{0}^{\tau_{D}} q(X(s)) d s\right) \varphi\left(X\left(\tau_{D}\right)\right)\right] \\
& +E_{x}^{0}\left[\int _ { 0 } ^ { \tau _ { D } } \operatorname { e x p } \left(\int_{0}^{t}\left\langle\widetilde{a}^{-1}(b-\hat{b})(X(s)), d M_{s}^{0}\right\rangle-\frac{1}{2} \int_{0}^{t}\left((b-\hat{b})^{*} \widetilde{a}^{-1}(b-\hat{b})\right)(X(s)) d s\right.\right. \\
& \left.\left.+N_{t}^{v}+\int_{0}^{t} q(X(s)) d s\right) F(X(t)) d t\right] .
\end{aligned}
$$

Example 4.3 If $q-J-\operatorname{div} \hat{b} \leq g$ for some $g \in L^{1}(D)$ in the sense of distribution and there exists a $x_{0} \in D$ such that

$$
\begin{gathered}
E_{x_{0}}^{0}\left[\operatorname { e x p } \left(\int_{0}^{\tau_{D}}\left\langle\widetilde{a}^{-1}(b-\hat{b})(X(s)), d M_{s}^{0}\right\rangle-\frac{1}{2} \int_{0}^{\tau_{D}}(b-\hat{b})^{*} \widetilde{a}^{-1}(b-\hat{b})(X(s)) d s\right.\right. \\
\left.\left.+\int_{0}^{\tau_{D}} g(X(s)) d s\right)\right]<\infty .
\end{gathered}
$$

Then condition (4.17) in Theorem 4.2 is satisfied.

Proof First we show that $P_{x_{0}}^{0}$-a.e., $N_{t}^{v}+\int_{0}^{t}(q-J)(X(s)) d s \leq \int_{0}^{t} g(X(s)) d s, \quad \forall t>0$.

Take an even, nonnegative function $\psi \in C_{0}^{\infty}\left(\mathbb{R}^{d}\right)$ such that $\int_{\mathbb{R}^{d}} \psi(x) d x=1$. Set $\psi_{k}(x):=k \psi(k x)$ and $\hat{b_{i}^{k}}(x):=\int_{\mathbb{R}^{d}} \hat{b}_{i}(y) \psi_{k}(x-y) d y$. Then $\hat{b_{i}^{k}} \in C_{0}^{\infty}\left(\mathbb{R}^{d}\right)$. Set $\left(g_{k}-q_{k}+J_{k}\right)(x):=\int_{\mathbb{R}^{d}}(g-q+J)(y) \psi_{k}(x-y) d y$. Then it's easy to see for any $x \in D$

$$
-\operatorname{div} \hat{b}^{k}(x) \leq\left(g_{k}-q_{k}+J_{k}\right)(x) .
$$

Set $f_{k}(x):=(g-q+J)(x)-\left(g_{k}-q_{k}+J_{k}\right)(x), P_{t}^{0} f_{k}(x):=E_{x}^{0}\left[f_{k}(X(s))\right]$. Let $\hat{P_{t}^{0}}$ be the dual operator of $P_{t}^{0}$ in $L^{2}(D)$. Since $g_{k}-q_{k}+J_{k}$ converges to $g-q+J$ in $L^{1}(D)$,

$$
\int_{D} E_{x}^{0}\left[\int_{0}^{t}\left|f_{k}\right|(X(s)) d s\right] d x=\int_{0}^{t}\left(P_{s}^{0} f_{k}, I_{D}\right) d s=\int_{0}^{t}\left(f_{k}, \hat{P_{s}^{0} I_{D}}\right) d s \leq t|| f_{k} \|_{L^{1}} \rightarrow 0
$$

as $k \rightarrow \infty$. Hence $\int_{0}^{t}\left|f_{k}\right|(X(s)) d s$ converges to 0 in $P_{m}^{0}$ where $P_{m}^{0}(\cdot)=\int_{D} P_{x}^{0}(\cdot) d x$. Then by the diagonal method there exists a subsequence $\left\{f_{k_{l}}\right\}$ such that $\int_{0}^{t}\left|f_{k_{l}}\right|(X(s)) d s$ converges to 0 uniformly in t on any compact set of $[0, \infty) P_{m}^{0}$-a.e.. For any $\delta \geq 0$, set

$$
\Lambda_{\delta}:=\left\{\omega: \text { for any } n \in \mathbb{N}, \lim _{k_{l} \rightarrow \infty} \int_{\delta}^{n}\left|f_{k_{l}}\right|(X(s, \omega)) d s=0\right\} .
$$

It's easy to show that $\Lambda_{0}=\theta_{\delta}\left(\Lambda_{\delta}\right)$. Set $h(x):=E_{x}^{0}\left[\Lambda_{0}\right]$. Then $h(x)=0$ m-a.e.. Since $\left(X_{t}, P_{x}^{0}\right)$ has a continuous transition density function, we have for any $x \in D$

$$
\begin{aligned}
P_{x}^{0}\left[\Lambda_{\delta}\right] & =E_{x}^{0}\left[I_{\Lambda_{0}}\left(\theta_{\delta}(\omega)\right)\right]=E_{x}^{0}\left[E_{x}^{0}\left[I_{\Lambda_{0}}\left(\theta_{\delta}(\omega)\right) \mid \mathcal{F}_{\delta}\right]\right] \\
& =E_{x}^{0}\left[E_{X(\delta)}^{0}\left[I_{\Lambda_{0}}\right]\right]=E_{x}^{0}[h(X(\delta))]=0, \forall \delta>0 .
\end{aligned}
$$


Set $\Lambda:=\left\{\omega:\right.$ for any $\left.n \in \mathbb{N}, \lim _{k_{l} \rightarrow \infty} \int_{\frac{1}{n}}^{n}\left|f_{k_{l}}\right|(X(s, \omega)) d s=0\right\}$. Then $\Lambda=\bigcap_{\delta>0} \Lambda_{\delta}$ and $P_{x}^{0}(\Lambda)=0$. Hence $P_{x}^{0}$-a.e.

$$
\lim _{k_{l} \rightarrow \infty} \int_{\frac{1}{n}}^{t}\left(g_{k_{l}}-q_{k_{l}}+J_{k_{l}}\right)(X(s)) d s=\int_{\frac{1}{n}}^{t}(g-q+J)(X(s)) d s, \quad \forall t>0, n \in \mathbb{N} .
$$

By Theorem 1 in [3] there exists a unique $v_{k} \in W_{0}^{1,2 p}(D)$ such that

$$
\int_{D}\left\langle a(x) \nabla v_{k}(x), \nabla \phi(x)\right\rangle d x=-2 \int_{D}\left\langle\hat{b}^{k}(x), \nabla \phi(x)\right\rangle d x, \quad \forall \phi \in W_{0}^{1,2}(D),
$$

where $\hat{b}^{k}=\left(\hat{b_{1}^{k}}, \hat{b_{2}^{k}}, \cdots, \hat{b_{d}^{k}}\right)^{*}$. Moreover

$$
\left\|\nabla v-\nabla v_{k}\right\|_{L^{2 p}} \leq \vartheta\left\|\hat{b}-\hat{b}^{k}\right\|_{L^{2 p}} .
$$

where $\vartheta$ is independent of $\hat{b}^{k}$. For every $k \geq 1$, we have the Fukushima's decomposition $v_{k}(X(t))-v_{k}(X(0))=M_{t}^{v_{k}}+N_{t}^{v_{k}} P_{x}^{0}$-a.e. for any $x \in D$ according to Theorem 1 in [11]. Note that for any $\phi \in W_{0}^{1,2}(D), \mathcal{E}^{0}\left(v_{k}, \phi\right)=\int_{D} \operatorname{div} \hat{b}^{k}(x) \phi(x) d x$ by (4.26). So $N_{t}^{v_{k}}=$ $-\int_{0}^{t} \operatorname{div} \hat{b}^{k}(X(s)) d s$. Since $|\hat{b}| \in L^{2 p}, \hat{b}^{k}=\left(\hat{b_{1}^{k}}, \hat{b_{2}^{k}}, \cdots, \hat{b_{d}^{k}}\right)$ converges to $\hat{b}$ in $L^{2 p}(D)$ as $k \rightarrow \infty$. Furthermore, by Sobolev embedding theorem, $\sup _{D}\left|v-v_{k}\right| \leq \vartheta_{0}|| \nabla v-$ $\nabla v_{k}\left\|_{L^{2 p}} \leq \vartheta_{0} \vartheta\right\| \hat{b}-\hat{b}^{k} \|_{L^{2 p}}$ where $\vartheta_{0}$ only depend on $d, p, D$. So for any $x \in D, v_{k}(X(t))$ converges to $v(X(t))$ uniformly on $[0, \infty) P_{x}^{0}$-a.e..

Replacing $E_{x}$ by $E_{x}^{0}$ in (3.6), we have the same estimate for some new constant $\sigma^{\prime \prime}$. By (1.3) and (4.27), for any $x \in D$,

$$
\begin{aligned}
E_{x}^{0}\left[\sup _{s \geq 0}\left(M_{s}^{v}-M_{s}^{v_{k}}\right)^{2}\right] & \leq 4 \sup _{s \geq 0} E_{x}^{0}\left[\left(M_{s}^{v}-M_{s}^{v_{k}}\right)^{2}\right] \\
& =4 E_{x}^{0}\left[\int_{0}^{\tau_{D}}\left\langle\tilde{a}\left(\nabla v-\nabla v_{k}\right)(X(s)),\left(\nabla v-\nabla v_{k}\right)(X(s))\right\rangle d s\right] \\
& \leq \frac{4}{\lambda} E_{x}^{0}\left[\int_{0}^{\tau_{D}}\left|\nabla v-\nabla v_{k}\right|^{2}(X(s)) d s\right] \\
& \leq \frac{4 \sigma^{\prime \prime}}{\lambda}\left\|\nabla v-\nabla v_{k}\right\|_{L^{2 p}}^{2} \leq \frac{4 \sigma^{\prime \prime} \vartheta^{2}}{\lambda}\left\|\hat{b}-\hat{b}^{k}\right\|_{L^{2 p}}^{2} \rightarrow 0
\end{aligned}
$$

as $k \rightarrow \infty$. So there exists a subsequence of $\left\{M_{t}^{v_{k_{l}}}\right\}_{l \geq 1}$ such that for any $x \in D$, it converges to $M_{t}^{v}$ uniformly on $[0, \infty) P_{x}^{0}$-a.e.. We still denote this subsequence by $\left\{M_{t}^{v_{k_{l}}}\right\}_{l \geq 1}$ for simplicity. Hence $N_{t}^{v_{k}}=-\int_{0}^{t} \operatorname{div} \hat{b}^{k_{l}}(X(s)) d s$ converges to $N_{t}^{v}$ uniformly on $[0, \infty) P_{x}^{0}$ a.e.. By (4.24)

$$
N_{t}^{v_{k_{l}}}-N_{\frac{1}{n}}^{v_{k_{l}}} \leq \int_{\frac{1}{n}}^{t}\left(g_{k_{l}}-q_{k_{l}}+J_{k_{l}}\right)(X(s)) d s .
$$

By (4.25) and (4.28), taking $k_{l} \rightarrow \infty$ and $n \rightarrow \infty$ in (4.28) we have $P_{x}^{0}$-a.e.

$$
N_{t}^{v} \leq \int_{0}^{t}(g-q+J)(X(s)) d s, \quad \forall t>0 .
$$

Thus we have

$$
\begin{aligned}
& E_{x_{0}}^{0}\left[\operatorname { e x p } \left(\int_{0}^{\tau_{D}}\left\langle\widetilde{a}^{-1}(b-\hat{b})(X(s)), d M_{s}^{0}\right\rangle-\frac{1}{2} \int_{0}^{\tau_{D}}(b-\hat{b})^{*} \widetilde{a}^{-1}(b-\hat{b})(X(s)) d s\right.\right. \\
& \left.\left.\quad+N_{\tau_{D}}^{v}+\int_{0}^{\tau_{D}}(q-J)(X(s)) d s\right)\right] \\
& \leq E_{x_{0}}^{0}\left[\operatorname { e x p } \left(\int_{0}^{\tau_{D}}\left\langle\widetilde{a}^{-1}(b-\hat{b})(X(s)), d M_{s}^{0}\right\rangle-\frac{1}{2} \int_{0}^{\tau_{D}}(b-\hat{b})^{*} \widetilde{a}^{-1}(b-\hat{b})(X(s)) d s\right.\right. \\
& \left.\left.\quad+\int_{0}^{\tau_{D}} g(X(s)) d s\right)\right]<\infty .
\end{aligned}
$$

Hence (4.17) is satisfied.

Acknowledgments This work is partly supported by National Natural Science Foundation of China (No.11671372, No.11431014, No.11401557). 
Open Access This article is distributed under the terms of the Creative Commons Attribution 4.0 International License (http://creativecommons.org/licenses/by/4.0/), which permits unrestricted use, distribution, and reproduction in any medium, provided you give appropriate credit to the original author(s) and the source, provide a link to the Creative Commons license, and indicate if changes were made.

\section{References}

1. Aronson, D.G.: Bounds for the fundamental solution of a parabolic equation. Bull. Amer. Math. Soc. 73(6), 890-896 (1967)

2. Aronson, D.G.: Non-negative solutions of linear parabolic equations. Annali della Scuola Normale Superiore di Pisa-Classe di Scienze 22(4), 607-694 (1968)

3. Auscher, P., Qafsaoui, M.: Observations on $w^{1, p}$ estimates for divergence elliptic equations with VMO coefficients. Bollettino della Unione Matematica Italiana-B (2), 487-510 (2002)

4. Briand, P., Delyon, B., Hu, Y., Pardoux, E., Stoica, L.: $L^{p}$ solutions of backward stochastic differential equations. Stoch. Process. Appl. 108(1), 109-129 (2003)

5. Chen, C.Z., Sun, W., Zhang, J.: Probabilistic representations of solutions of elliptic boundary value problem and non-symmetric semigroups. J. Differ. Equat. 260(1), 26-55 (2016)

6. Chen, Z.Q., Song, R.: General gauge and conditional gauge theorems. Ann. Probab. 1313-1339 (2002)

7. Chen, Z.Q., Zhang, T.: Time-reversal and elliptic boundary value problems. Ann. Probab. 1008-1043 (2009)

8. Chen, Z.Q., Zhao, Z.: Diffusion processes and second order elliptic operators with singular coefficients for lower order terms. Math. Ann. 302(1), 323-357 (1995)

9. Chung, K.L., Zhao, Z.: From Brownian motion to schrodingers equation. Springer Science \& Business Media (2012)

10. Dellacherie, C., Meyer, P.A.: Probabilities and potential. B, volume 72 of North-Holland Mathematics Studies (1982)

11. Fukushima, M.: On a Decomposition of Additive Functionals in the Strict Sense for a Symmetric Markov Process. In: Dirichlet Forms and Stochastic Processes (Beijing, 1993), pp. 155-169. Walter de Gruyter, Berlin (1995)

12. Kakutani, S.: Two-dimensional Brownian Motion and Harmonic Functions. Proceedings of the Imperial Academy 20(10), 706-714 (1944)

13. Lunt, J., Lyons, T.J., Zhang, T.: Integrability of functionals of Dirichlet processes, probabilistic representations of semigroups, and estimates of heat kernels. J. Funct. Anal. 153(2), 320-342 (1998)

14. Ma, Z.M., Rockner, M.: Introduction to the theory of (non-symmetric) Dirichlet forms. Springer Science \& Business Media (2012)

15. Oshima, Y.: Semi-dirichlet forms and Markov processes. Walter de Gruyter (2013)

16. Pardoux, E., Peng, S.: Backward stochastic differential equations and quasilinear parabolic partial differential equations. In: Stochastic Partial Differential Equations and Their Applications (Lect. Notes Control Inf. Sci. 176), pp. 200-217. Springer, Berlin (1992)

17. Peng, S.: Probabilistic interpretation for systems of quasilinear parabolic partial differential equations. Stochast. Stochast. Rep. 37(1-2), 61-74 (1991)

18. Zhang, T.: A probabilistic approach to Dirichlet problems of semilinear elliptic PDEs with singular coefficients. Ann. Probab. 39(4), 1502-1527 (2011) 